\title{
Cholinergic, GABAergic, and Glutamate-Enriched Inputs from the Mesopontine Tegmentum to the Subthalamic Nucleus in the Rat
}

\author{
M. D. Bevan and J. P. Bolam \\ MRC Anatomical Neuropharmacology Unit and Department of Pharmacology, Oxford OX1 3TH, United Kingdom
}

In order to clarify the origin and to examine the neurochemistry and synaptology of the projection from the mesopontine tegmentum (MTg) to the subthalamic nucleus (STN), rats received discrete deposits of anterograde tracers in different regions of the MTg. Anterogradely labeled fibers were examined in the light and electron microscopes. The distribution of GABA or glutamate immunoreactivity was examined by post-embedding immunocytochemistry.

The anterograde tracing demonstrated that the projection to the STN arises from at least three divisions of the MTg: the area defined by the cholinergic neurons of the pedunculopontine region (PPN-Ch 5), the more medial and largely noncholinergic midbrain extrapyramidal area (MEA) and to a lesser extent the laterodorsal tegmental nucleus (LDTg). Post-embedding immunocytochemistry revealed that there are GABA-immunopositive and immunonegative components to this projection and at least a proportion of the GABA-immunonegative component is enriched in glutamate immunoreactivity. The similarity of the morphology, trajectory and synaptology of the anterogradely labeled fibers and the choline acetyltransferase (ChAT)-immunopositive fibers supports the proposal that at least part of the projection is cholinergic.

The terminals anterogradely labeled from the MTg and the ChAT-immunoreactive terminals form asymmetrical synapses with the dendrites and spines of subthalamic neurons. Both anterogradely labeled and ChAT-positive terminals make convergent synaptic contacts with GABAimmunoreactive terminals that form symmetrical synaptic contacts and are probably derived from the globus pallidus. Taken together these findings imply that the MTg sends cholinergic, GABAergic and glutamatergic projections to the STN where at least one of the functional roles is to modulate the indirect pathway of information flow through the basal ganglia that is carried via the pallidosubthalamic projection.

[Key words: ACh, basal ganglia, BDA, ChAT, GABA, glutamate, laterodorsal tegmental nucleus, mesopontine teg-

\footnotetext{
Received Apr. 24, 1995; revised June 16, 1995; accepted June 22, 1995.

We thank Caroline Francis, Paul Jays, Frank Kennedy, and Liz Norman for technical assistance, Alan Levey for advice on the anterograde tracing study, and Peter Somogyi for the GABA antiserum and guidance throughout the postembedding imunocytochemical cxperiments. This work was funded by the Wellcome Trust and the Medical Research Council, U.K.

Correspondence should be addressed to Mark Bevan, MRC Anatomical Neuropharmacology Unit and Department of Pharmacology, Mansfield Road, Oxford OX1 3TH, United Kingdom.

Copyright $\mathrm{C} 1995$ Society for Neuroscience $0270-6474 / 95 / 157105-16 \$ 05.00 / 0$
}

mentum, midbrain extrapyramidal area, pedunculopontine nucleus, PHA-L, subthalamic nucleus]

Neurons of the subthalamic nucleus exert a key role in the control of movement by virtue of their glutamatergic innervation of the basal ganglia output nuclei that in turn project to the thalamus and subcortical premotor areas (Kita and Kitai, 1987: Nakanishi et al., 1987; Smith and Parent, 1988; Groenewegen and Berendse, 1990; Smith et al., 1990a; Nakanishi et al., 1991; Rinvik and Ottersen, 1993; Bevan et al., 1994a,b; Smith et al., 1994a). The activity of subthalamic neurons is thus important in the manifestation of both normal movement (Matsumara et al., 1992; Wichmann et al., 1994a) and abnormal movement in neurological disorders of great clinical significance, for example, Parkinson's disease, chorea, and hemiballism (Crossman, 1987; DeLong, 1990; Filion and Tremblay, 1991; Mitchell et al., 1992; Bergman et al., 1994; Wichmann et al., 1994b). The recent development of therapies for the treatment of Parkinson's disease which act by reducing the influence of an abnormally overactive STN (Bergman et al., 1990; Aziz et al., 1991; Brotchic ct al., 1991; Benazzouz et al., 1993) has prompted us to reexamine the origin, synaptic nature, and neurochemistry of the major inputs to the STN (Bevan et al., 1995). The subject of the present study is the input to the STN derived from the MTg (Bowsher, 1975; Graybiel, 1977; Nomura et al., 1980; Saper and Loewy, 1982; Jackson and Crossman, 1983; Moon-Edley and Graybiel, 1983; Woolf and Butcher, 1986; Moriizumi et al., 1987; Lee et al., 1988; Spann and Grofova, 1989; Canteras et al., 1990; Lavoie and Parent, 1994a). The MTg has been reported to be involved in a multitude of functions including locomotion, sleep-wake mechanisms and arousal (see Garcia-Rill, 1991, and Jones, 1993, for reviews) and its projection to the STN is one route through which this region could exert profound effects on movement. The exact origin and neurochemical nature of the mesopontine tegmental projection to the STN, and indeed to the basal ganglia in general, have been the subject of much debate (Woolf and Butcher, 1986; Rye et al., 1987; Lee et al., 1988; Spann and Grofova, 1989). It has been proposed that this projection arises almost exclusively from the cholinergic neurons of the pedunculopontine nucleus and the laterodorsal tegmental nucleus, or almost exclusively from the noncholinergic neurons of the MEA or from both sites (Woolf and Butcher, 1986; Lee et al., 1988; Spann and Grofova, 1989). The first objective of the present study was therefore to reexamine the site(s) of origin of the projection hy placing small deposits of anterograde tracers in different parts of the MTg. The exact location of these deposits was defined with respect to cholinergic neurons in the MTg, visualized by NADPH-diaphorase histochemistry (Vincent et al., 1983). 
Since it has been proposed that the cholinergic innervation of the STN is derived from the MTg (Woolf and Butcher, 1986; Mesulam et al., 1992), the second objective of this study was to compare the morphology of axons and the synaptic nature of terminals that are immunoreactive for ChAT with those anterogradely labeled from the $\mathrm{MTg}$.

On the basis of immunocytochemistry for glutamate or GABA several populations of neurons have been observed in the M'I'g (Clements and Grant, 1990; Jones, 1990, 1993; Lavoie and Parent, $1994 \mathrm{~b}, \mathrm{c})$. Ilowever the projection characteristics of the amino acid-positive and -negative neurons have largely been unstudied (Lavoie and Parent, 1994c). In view of the importance of GABA and glutamate in neurotransmission, the third objective of this study was to determine the amino acid content of the boutons and synaptic terminals in the STN that were derived from the MTg. This issue was addressed by combining anterograde tracing or immunocytochemistry for ChAT and post-entbedding immunocytochemistry for glutamate and/or GABA.

A major pathway of information flow through the basal ganglia is the pathway cortex-neostriatum-globus pallidus-STNbasal ganglia output nuclei (Albin et al., 1989; Tremblay and Filion, 1989; Alexander and Crutcher, 1990). The physiological and pathophysiological actions of this "indirect pathway" are associated with the increased inhibition of basal ganglia targets and hence the suppression of movement (Crossman, 1987; Millcr and DeLong, 1987; Albin et al., 1989; DeLong, 1990; Matsumara et al., 1992; Bergman et al., 1994; Wichmann et al., 1994a,b). The fourth and final objective of this study was therefore to determine whether synaptic terminals derived from the MTg are in a position to modulate the flow of information through this pathway by making convergent synaptic contact with subthalamic neurons that also receive synaptic input from the globus pallidus. Terminals derived from the MTg were anterogradely or immunocytochemically labeled and those from the globus pallidus were defined on the basis of ultrastructural and immunocytochemical criteria (Smith et al., 1990b).

\section{Materials and Methods}

Preparation of tissue. All surgical procedures were carried out on male Sprague-Dawley rats (250-350 gm) under deep anaesthesia, which was induced and maintained by intra-peritoneal injection of pentobarbitone. Injections of neuronal tracers were placed into specific regions of the MTg under stereotaxic guidance using coordinates derived from the atlas of Paxinos and Watson (1986).

Phaseolus vulgaris-leucoagglutinin (PHA-L, 2.5\% in $0.01 \mathrm{M}$ phosphate buffer, pH 8.0; Vector Laboratories, Peterborough, U.K.) (Gerfen and Sawchenko, 1984) and biotinylated dextran amine (BDA, $10 \%$ in $0.9 \%$ sodium chloride, Molecular Probes) (Veenman et al., 1992) were delivered by iontophoresis into the MTg via glass micropipettes of 10 $35 \mu \mathrm{m}$ (I.D.) using a pulsed (7 sec on, $7 \mathrm{sec}$ off) cathodal current (5$9 \mu \mathrm{A})$ over $10-20 \mathrm{~min}$.

Animals that received injections of neuronal tracers were perfused after 4-8 d. These animals, together with unoperated animals for choline acetyltransferase (ChAT) immunocytochemistry, were deeply anaesthetized and solutions were perfused via the ascending aorta using a peristaltic pump. Prior to perfusion with fixative, the blood was removed by rapid perfusion with $100 \mathrm{ml}$ of phosphate-buffered saline (PBS) over 1-2 min. Animals for the visualization of PHA-L or ChAT-immunoreactivities were then perfused with $200 \mathrm{ml}$ of $0.1 \%$ to $0.5 \%$ glutaraldehyde and $3 \%$ paraformaldehyde in phosphate buffer $(\mathrm{PB} ; 0.1 \mathrm{M}, \mathrm{pH}$ 7.4) over $5 \mathrm{~min}$, followed by $100 \mathrm{ml}$ of $3 \%$ paraformaldehyde in $\mathrm{PB}$ over $10 \mathrm{~min}$. Animals for the visualization of BDA were perfused with $300 \mathrm{ml}$ of $1.5 \%$ glutaraldehyde and $2 \%$ paraformaldehyde in phosphate buffer (PB; $0.1 \mathrm{M}, \mathrm{pH} 7.4$ ) over $20 \mathrm{~min}$. Following fixation, the brain was removed from the cranium, divided into $5 \mathrm{~mm}$ thick coronal slices and stored in phosphate-buffered saline (PBS) at $4^{\circ} \mathrm{C}$ prior to further processing. From each slice $60 \mu \mathrm{m}$ coronal sections of the injection sites and the STN were taken using a vibrating microtome and collected in PBS. Sections from animals that had been fixed with concentrations of 0.5 or $1.5 \%$ glutaraldehyde were then pretreated with $1 \%$ sodium borohydride in distilled water for 5-20 min. These sections were then washed many times in PBS prior to further processing. All sections were freeze-thawed as described previously (von Krosigk and Smith, 1991).

Visualization of neuronal tracers. The BDA was visualized by incubation of the sections in an avidin-biotin-peroxidase complex (ABC; 1:100 dilution; Vector Laboratories, Peterborough. U.K.) in PBS containing $1 \%$ bovine serum albumin overnight at room temperature or 48 hr at $4^{\circ} \mathrm{C}$. Peroxidase linked to BDA by the avidin-biotin bridge was revealed by placing the sections in Tris buffer $(0.05 \mathrm{M}, \mathrm{pH} 7.4)$ containing $0.025 \% \mathrm{DAB}$ and $0.006 \%$ hydrogen peroxide for $10-15 \mathrm{~min}$. The reaction was terminated by rinsing several times in Tris buffer.

The PHA- $\mathrm{L}$ was visualized by incubating the sections in rabbit antiPHA-L antibody (1:1000 dilution; Dakopatts, Netherlands) overnight at room temperature or $48 \mathrm{hr}$ at $4^{\circ} \mathrm{C}$. After many washes in $\mathrm{PBS}$, the sections were incubated in a solution of biotinylated goat anti-rabbit IgG (1:200; Vector Laboratories, Peterborough, U.K.) at room temperature for $2 \mathrm{hr}$ followed by a $2 \mathrm{hr}$ incubation in $\mathrm{ABC}(1: 100)$. Bound peroxidase was revealed using DAB as above. The antisera were diluted in PBS containing $1 \%$ bovine serum albumin and $2 \%$ normal goat serum.

Immunocytochemistry for choline acetyltransferase. Sections were incubated in monoclonal antibody to choline acetyltransferase which was raised in rat-mouse hybrid cells $(1: 250$, Bochringer Mannheim, Germany) overnight at room temperature or $48 \mathrm{hr}$ at $4^{\circ} \mathrm{C}$. After several washes in PBS the sections were incubated in a solution of biotinylated goat anti-rat IgG (1:200; Vector Laboratories. Peterborough, U.K.) for $2 \mathrm{hr}$ at room temperature, followed by a $2 \mathrm{hr}$ incubation in ABC. Bound peroxidase was revealed using DAB as above. The antisera were diluted in PBS containing $2 \%$ bovine serum albumin and $2 \%$ normal goat serum.

Histochemistry for NADPH-diaphorase. Following the visualization of the neuronal tracers, sections of the MTg were processed for the visualization of NADPH diaphorase-positive neurons. These sections were incubated in a solution containing $0.1 \%$ NADPH (Sigma, U.K.), $0.1 \%$ nitro blue tetrazolium (Sigma, U.K.) and $0.3 \%$ Triton X-100 dissolved in Tris $\mathrm{HCl}\left(0.05 \mathrm{M}, \mathrm{pH} \mathrm{7.4)}\right.$ at $37^{\circ} \mathrm{C}$ for $5-30 \mathrm{~min}$ (Vincent et al., 1983). The reaction was terminated by rinsing the sections several times in Tris buffer $(0.05 \mathrm{M}, \mathrm{pH} 7.4)$. These sections were then mounted on gelatin-coated slides and processed for standard light microscopy.

Processing of sections for correlated light and electron microscopy. Sections through the STN were placed flat at the bottom of a petri dish and postfixed in $1 \%$ osmium tetroxide (Oxchem, U.K.) (in $0.1 \mathrm{M} \mathrm{PB}$ at $\mathrm{pH}$ 7.4). The sections were then washed in phosphate buffer and rapidly dehydrated through a graded series of alcohols. To enhance the contrast of the tissue in the electron microscope, the sections were stained with $1 \%$ uranyl acetate (Taab, U.K.) at the $70 \%$ alcohol phase. The sections were washed twice for $10 \mathrm{~min}$ in propylene oxide and placed in resin (Durcupan, Fluka, U.K.) overnight. Finally, the sections were embedded in resin on microscope slides, placed in an oven and cured for $48 \mathrm{hr}$ at $60^{\circ} \mathrm{C}$.

Analysis of material. The injection sites in the MTg and the distribution of NADPH diaphorase-positive neurons were analyzed in the light microscope and drawn with the aid of a drawing tube. The density of anterograde labeling in the STN was assessed qualitatively from three sections taken from the rostral, middle and caudal thirds of the ipsilateral nucleus of each animal. A simple rating scale was used to score the density of anterograde transport in each section: $0=$ no anterograde labeling, 1 = sparse anterograde labeling, 2 = moderate anterograde labcling, $3=$ dense anterograde labeling. The STN from some of these sections and sections that had been immunolabeled for ChAT were drawn and/or photographed and selected areas that were rich in labeled structures were cut out from the slides and glued to blank resin blocks. Serial ultrathin sections were cut on a Reichert-Jung Ultracut-E ultramicrotome and collected on Pioloform-coated copper slot grids. The ultrathin sections were contrasted with lead citrate for 1-2 min and examined in a Phillips CM10 electron microscope.

To compare the morphology and the nature of the postsynaptic targets of terminals anterogradely labeled from the mesopontine tegmental region and terminals immunopositive for ChAT, all parts of ultrathin sections taken from regions that were dense in anterogradely labeled structures were scanned at a constant magnification. All labeled terminals forming synaptic contacts were counted and classified according to their 
origin, morphology and the nature of the postsynaptic target. Crosssectional areas of labeled terminals and their synaptic targets were determined from photographs using a digitizing pad and MacStereology software.

Postembedding immunocytochemistry for GABA and glutamate. In order to test for the presence of fixed glutamate and GABA in anterogradely labeled and nonlabeled terminals in the STN, ultrathin sections were collected on gold grids and labeled by the post-embedding immunogold method. Glutamate-like and GABA-like immunoreactivity (hereafter referred to as glutamate and GABA immunoreactivity, respectively) were detected using polyclonal antisera directed against fixed amino acid-protein complexes.

Adjacent sections were immunolabeled for glutamate and GABA using a slight modification of the method described by Phend and coworkers (1992). The grids were first washed in $0.05 \mathrm{M}$ Tris buffer $(\mathrm{pH} 7.6)$ containing $0.9 \% \mathrm{NaCl}$ and $0.01 \%$ Triton $\mathrm{X}-100$ (TBS-Triton) and then incubated overnight at room temperature on drops of a 1:5,000 dilution of rabbit anti-glutamate antiserum (Arnel Products Co., New York; Hepler et al., 1988; Petrusz et al., 1990; Abdullah et al., 1992) or a 1:15,000 dilution of rabbit anti-GABA antiserum (code 9; Hodgson et al., 1985; Somogyi and Hodgson, 1985; Somogyi et al., 1985) in TBS-Triton. After several washes in TBS-Triton and one wash in TBS at $\mathrm{pH} 8.2$, the grids were incubated for $1-1.5 \mathrm{hr}$ at room temperature in a 1:25 dilution of 15 $\mathrm{nm}$ gold-conjugated goat anti-rabbit IgG (BioCell, Cardiff, U.K.) in TBS at $\mathrm{pH}$ 8.2. The grids were washed in TBS at $\mathrm{pH} 8.2$ and then in water, stained with $1 \%$ aqueous uranyl acetate for $1-1.5 \mathrm{hr}$ and then with lead citrate. The sections were then examined in the electron microscope.

Analysis and quantification of glutamate-and GABA-immunolabeled marerial. Inmunoreactivity for glutamate or GABA was detected by the presence of the electron dense immunogold particles overlying labeled structures. In order to quantify the immunoreactivity, the density (particles $/ \mu \mathrm{m}^{2}$ ) of immunogold particles overlying individual structures was calculated. Although glutamate is ubiquitous, it has been demonstrated that putative glutamatergic nerve terminals are enriched in glutamate immunoreactivity compared to GABAergic terminals (Somogyi et al., 1986; Ottersen, 1989; Ottersen and Storm-Mathisen, 1989; Llewellyn-Smith et al., 1992; Phend et al., 1992; Valtschanoff et al., 1994; Bevan et al., 1995). In the STN all terminals forming symmetrical synapses are immunoreactive for GABA (Bevan et al., 1995). The density of immunogold particles overlying a glutamate-enriched structure was therefore expressed as a ratio of that overlying terminals forming symmetrical synapses. In order to control for the variability in immunolabeling on different days, between grids on the same run, or even between sections on the same grid, the data were normalized by using the immunogold particle density overlying terminals forming symmetrical synapses in the same section.

Since GABA is only found in high concentrations in structures which use it as a neurotransmitter, the pattern of GABA immunoreactivity in the STN was clearer than that of glutamate immunoreactivity. A structure was deemed to be immunopositive for GABA when the labeling density of that structure was at least 10 (tissue that had been perfused with fixative containing $1.5 \%$ glutaraldehyde) or six (tissue that had been perfused with fixative containing $0.3-0.5 \%$ glutaraldehyde) times greater than background labeling on the same ultrathin section. Background GABA labeling was defined as the density of immunoparticles overlying perikarya and dendrites as subthalamic neurons use glutamate and not GABA as their neurotransmitter (Smith and Parent, 1988). As with glutamate immunolabeling, GABA immunoreactivity was normalized with respect to dendrites and cell bodies from the same ultrathin section. The density of labeling for glutamate and GABA was corrected for nonspecific binding of the antibodies to tissue-free resin by subtracting the density of gold particles overlying the lumen of capillaries in the same ultrathin section.

Synaptic terminals and anterogradely labeled boutons or terminals were selected for analysis randomly by systematically scanning the glutamate-immunolabeled ultrathin sections; each synaptic terminal or anterogradely labeled bouton or synaptic terminal that was encountered was photographed and the density of immunogold particles overlying them was calculated. The values were then corrected and normalized as described above. Each of these structures, as well as some terminals forming symmetrical synapses were then examined in the adjacent GABA-labeled section. The glutamate and GABA immunoreactivity of populations of boutons were compared statistically using the MannWhitney $U$ test. $P$-Values that were less than 0.05 following Bonferoni's correction for multiple testing were considered to be significant.
Another search strategy was adopted to determine whether individual subthalamic neurons received convergent synaptic contact from cholinergic or mesopontine tegmental terminals and GABA-immunopositive terminals. For this analysis long series of ultrathin sections were taken and then all the sections were immunolabeled for GABA. These were then systematically scanned in the electron microscope for convergent synaptic contacts. The GABA-immunoreactivity of these terminals was assessed quantitatively as described above.

\section{Results}

\section{Light microscopic analysis}

Injection sites in the MTg. Eleven BDA injections (Figs. $1 B-D$, $2 A-K$ ) and 4 PHA-L injections (Figs. $1 A, 2 L-O$ ) were made in the MTg. The injection sites were characterized by densely filled neurons, although the clarity and extent of the cell filling was greater with BDA (Fig. $1 B-D$ ). In addition a halo of diffuse extracellular staining surrounded the PHA-L labeled neurons (Fig. 1A). The appcarance of BDA- and PHA-L-labcled ncurons and their processes were similar, they were filled in Golgi-fashion with the brown $\mathrm{DAB}$ reaction product that was used to visualize the tracers (Fig. 1). The neuronal tracers were easily distinguishable from NADPH diaphorase-positive neurons (cholinergic neurons) that were identified by the blue-purple reaction product that labeled their proximal regions (Fig. 1).

The injection sites could be roughly divided into four groups. (1) Injections largely confined to the PPN-Ch 5 (Butcher and Semba, 1989) (PPN-Ch 5, $n=6$ ), three of which involved the lateral half of the PPN-Ch 5 (and the adjacent lateral lemniscus) (Fig. $2 C-E$ ) and three included the majority of the PPN-Ch 5 (Figs. $1 B, 2 A, B, F$ ). (2) Injections placed in the MEA and thus largely avoiding the PPN-Ch $5(n=3)$ (Figs. $1 C, 2 G, N, O)$. (3) Injections involving both the PPN-Ch 5 and the MEA $(n=4)$ (Figs. $1 A, 2 H, I, L, M$ ). (4) Injections in the LDTg Ch 6 (Butcher and Semba, 1989) $(n-2$; Figs. $1 D, 2 J, K)$.

The mesopontine tegmental projection to the subthalamic nucleus. Each of the four classes of injection led to anterograde labeling in the ipsilateral and contralateral subthalamic nuclei (Fig. $3 B-G$ ), although the density of labeling was always far greater in the ipsilateral STN. The morphology of the anterogradely labeled fibers and their position in the STN was not clearly related to the site of injection. They ranged from thin fibers with small varicosities through to thick fibers with large varicosities. In addition some fibers coursed in simple linear fashion across large expanses of the STN whereas others followed very tortuous paths and were restricted to much smaller areas of the nucleus.

The density of anterograde labeling in the STN was mainly related to the site of the injection in the mesopontine tegmental region rather than the size of injection (Fig. 2, Table 1). In group 1 , the laterally located injections led to sparse labeling whereas the more medially located injections led to moderate or dense labeling. In groups 2 and 3, the injections resulted in moderate to dense labeling and in group 4 the injections led to very sparse labeling.

Cholinergic innervation of the subthalamic nucleus. Cholinergic fibers were identified by immunocytochemistry for ChAT visualized by the brown $\mathrm{DAB}$ reaction product. They were present throughout the STN with no obvious differences in density between different regions of the nucleus. Like the anterogradely labeled fibers they also displayed a variety of morphologies and trajectories (Fig. 3A). The majority of the varicosities were the en passant type, although some terminal varicosities were also observed. 


\section{Electron microscopic analysis}

Anterogradely labeled- and ChAT-immunopositive structures. The electron microscopic analysis was carried out on tissue from two animals (Fig. $2 L, M$ ) that had received large injections of PHA-L that included both the PPN-Ch 5 and the MEA and two animals that were used solely for ChAT immunocytochemistry. The animals were perfused with the same fixative and their tissue treated in a similar manner so that comparisons of the sizes of the terminals and their postsynaptic targets could be made. Labeled structures were identified by the presence of the electron dense DAB reaction product that adhered to the outer surfaces of organelles and the inner surface of plasma membranes. Reaction product was found only in nerve terminals and myelinated and unmyelinated axons.

The ultrastructural characteristics of anterogradely labeled terminals and ChAT immunopositive terminals and their postsynaptic targets were similar (Fig. 4). Terminals anterogradely labeled from the MTg (Fig. 4A,B) were of small to medium size (cross-sectional area at the level of the synapse: range, 0.65$0.712 \mu \mathrm{m}^{2}$; mean $\pm \mathrm{SEM}, 0.323 \pm 0.02 \mu \mathrm{m}^{2}, n=68$ ), were densely packed or loosely packed with round or pleomorphic synaptic vesicles and made asymmetrical synaptic contacts with dendritic shafts or spines (cross-sectional area of postsynaptic

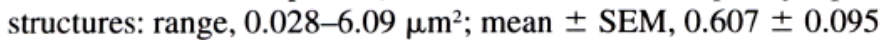
$\mu \mathrm{m}^{2}, n=69$ ), (Fig. 4A,B). Terminals immunoreactive for ChAT were also of small to medium size (cross-sectional area at the level of the synapse: range, 0.051-0.697 $\mu \mathrm{m}^{2}$; mean \pm SEM, $0.251 \pm 0.014 \mu \mathrm{m}^{2}, n=98$ ), were densely packed or loosely packed with round or pleomorphic synaptic vesicles and made asymmetrical synaptic contacts with dendritic shafts or spines (cross-sectional area of postsynaptic structures: range, 0.013$1.926 \mu \mathrm{m}^{2}$; mean \pm SEM, $0.420 \pm 0.037 \mu \mathrm{m}^{2}, n=92$ ) (Fig. $4 C, D)$. The size of the anterogradely labeled terminals was significantly greater than the ChAT-immunopositive terminals (Mann-Whitney $U$ test, $p<0.002$ ). There was no significant difference in the size of the postsynaptic targets of the anterogradely labeled terminals and the ChAT-immunopositive terminals.

Glutamate and GABA immunoreactivity in the STN. Postembedding immunocytochemistry for glutamate and GABA was carried out on sections from eight animals that had received BDA injections and had been perfused with fixative solution that contained $1.5 \%$ glutaraldehyde. Three of these animals had received injections in the MTg (Fig. $2 B, G, H$ ) and five had received tracer deposits in the cortex or the thalamus as part of a separate study (Bevan et al., 1995). In the present study only nonanterogradely labeled boutons from the latter five animals were included in the analysis. The pattern of glutamate and

Figure 1. $A-D$, Light micrographs of deposits of PHA-L $(A)$ and BDA $(B, C, D)$ in the MTg. The neurons that incorporated these tracers were visualized with $\mathrm{DAB}$ giving them a brown appearance. Cholinergic neurons in this region are defined by the presence of the blue-purple reaction product formed by the NADPH-diaphorase. $A, \mathrm{~A}$ large injection of PHA-L in the MTg involving both the PPN-Ch 5 and the MEA (corresponds to Fig. $2 L$ ). B, A more discrete injection of BDA in the PPN-Ch5 which largely avoids the adjacent MEA (corresponds to Fig. $2 B$ ). $C$, An injection of BDA in the MEA which largely avoids the adjacent PPN-Ch 5 (corresponds to Fig. $2 G$ ). $D$, An injection of BDA which is largely confined to the LDTg-Ch 6 (corresponds to Fig. $2 K$ ). $L D T g$, Laterodorsal tegmental region. All micrographs are at the same magnification; bar in $A=500 \mu \mathrm{m}$.
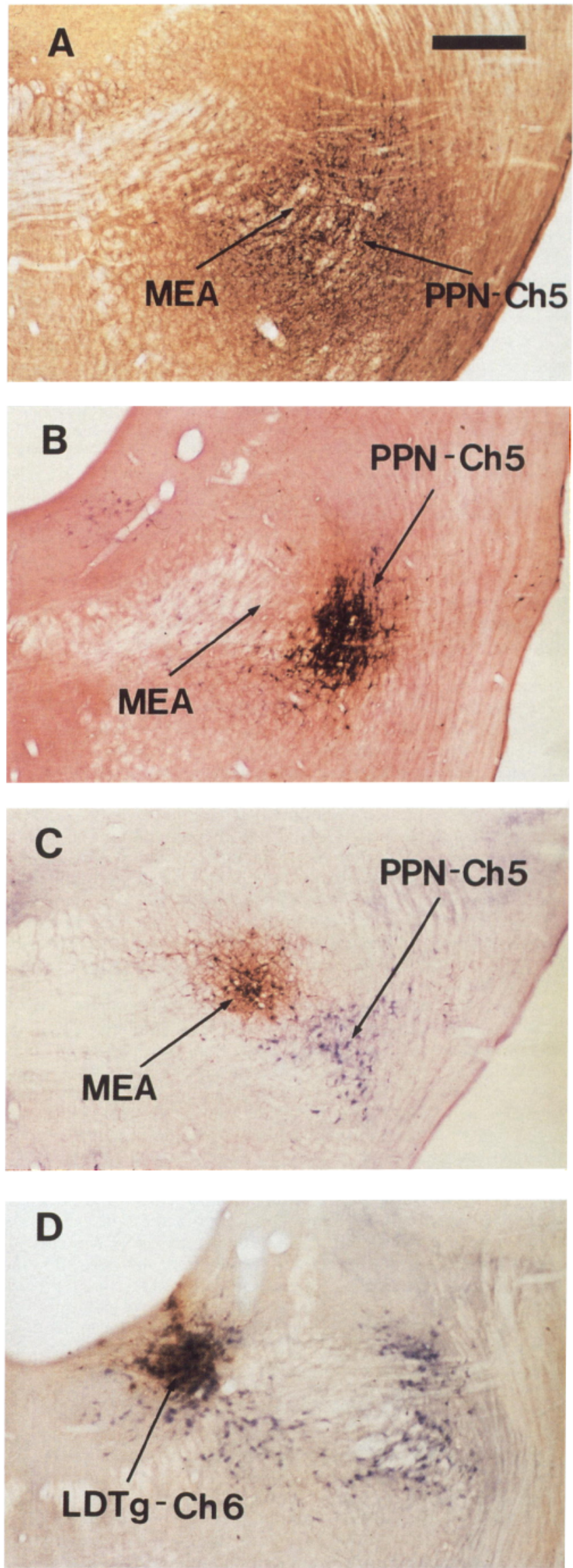

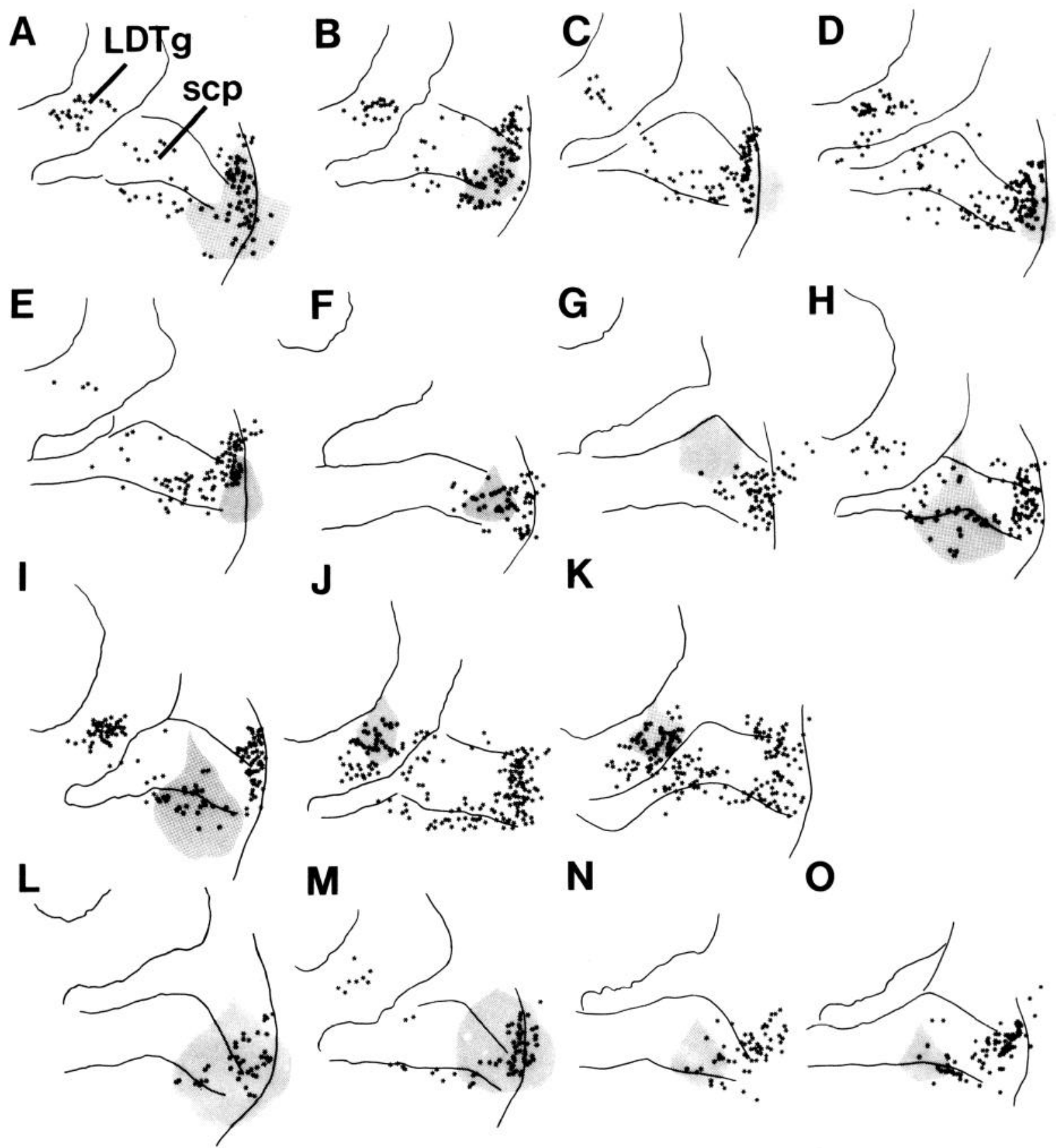

Figure 2. $A-O$, Schematic diagrams demonstrating the sites of 15 tracer deposits $(A-K, \mathrm{BDA} ; L-O, \mathrm{PHA}-\mathrm{L})($ shaded area $)$ in the MTg in relation to NADPH-diaphorase-positive (cholinergic) neurons which are denoted by asterisks. The injection sites can divided into four groups: (1) injections largely confined to the PPN-Ch $5(n=6)$ of which three injections involved the lateral half of the PPN-Ch 5 (and the adjacent lateral lemniscus) (animals $C-E$ ) and three injections included the majority of the medial and laterally located PPN-Ch $5(A, B, F)$; $(2)$ injections placed in the MEA and largely avoiding the PPN-Ch $5(G, N, O)$; (3) injections involving both the PPN-Ch 5 and the MEA $(H, I, L, M)$ : (4) injections in the LDTgCh $6(J, K)$. Abbreviations: $L D T g$, laterodorsal tegmental region; scp, superior cerebellar peduncle.

GABA immunolabeling in the STN was similar for all eight animals. The glutamate and GABA labeling of neuronal elements in the STN has been reported in detail previously (Bevan et al., 1995) and therefore will only be described in brief. Relatively high levels of glutamate immunoreactivity were associated with the perikarya, dendrites and spines of subthalamic neurons (see Figs. $6 C, 8 A, C, E$ ). High levels were also associated with the majority of terminals forming asymmetrical synaptic contacts. This labeling was consistently higher than that associated with terminals forming symmetrical synaptic contacts. Immunoreactivity for GABA was detected in all terminals forming symmetrical synaptic contacts (Figs. 5, 6A, 9, 10) and a small proportion of the terminals forming asymmetrical synaptic contacts (Figs. 5, 6A). The immunoreactivity associated with several populations of terminals, defined on the basis of their origin or morphology was analyzed quantitatively.

(1) Nonanterogradely labeled terminals. Terminals forming asymmetrical synaptic contacts which were immunonegative for GABA, were significantly $(p<0.001$, Mann-Whitney $U$ test $)$ enriched in glutamate immunoreactivity (mean \pm SEM of index of glutamate immunoreactivity, $2.693 \pm 0.127, n=36$ ) compared to that found in terminals forming symmetrical synapses 

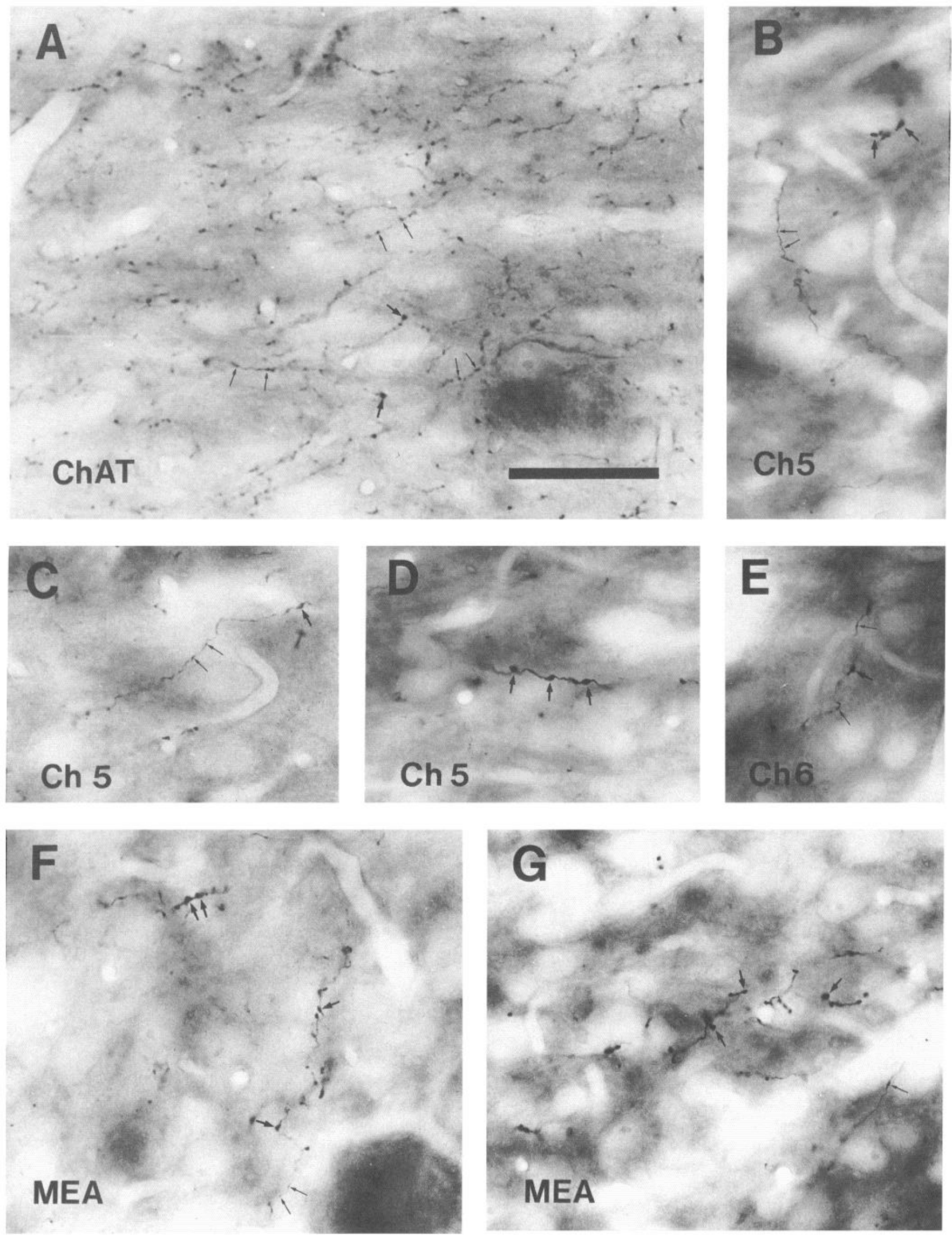


\begin{tabular}{|c|c|c|c|c|}
\hline $\begin{array}{l}\text { Injection } \\
\text { group }\end{array}$ & $\begin{array}{l}\text { Injection } \\
\text { site } \\
\text { (Fig. 2) }\end{array}$ & $\begin{array}{l}\text { Labeling } \\
\text { subthala } \\
\text { Caudal }\end{array}$ & $\begin{array}{l}\text { gin in } \\
\text { amic nuc } \\
\text { Middle }\end{array}$ & $\begin{array}{l}\text { Rostral } \\
\text { Ross }\end{array}$ \\
\hline \multirow[t]{3}{*}{ PPN-Ch 5 center } & $\mathrm{A}$ & 2 & 1 & 1 \\
\hline & $\mathrm{B}$ & 3 & 3 & 3 \\
\hline & $\mathrm{F}$ & 2 & 2 & 2 \\
\hline \multirow[t]{3}{*}{ PPN-Ch5 lateral } & $\mathrm{C}$ & 2 & 1 & 2 \\
\hline & $\mathrm{D}$ & 1 & 1 & 1 \\
\hline & $\mathrm{E}$ & 1 & 1 & 1 \\
\hline \multirow[t]{3}{*}{ MEA } & $\mathrm{G}$ & 3 & 2 & 2 \\
\hline & $N$ & 2 & 2 & 2 \\
\hline & $\mathrm{O}$ & 1 & 1 & 1 \\
\hline \multirow[t]{4}{*}{ PPN-Ch 5 plus MEA } & $\mathbf{H}$ & 2 & 3 & 3 \\
\hline & I & 2 & 3 & 3 \\
\hline & $\mathrm{L}$ & 3 & 3 & 3 \\
\hline & M & 1 & 2 & 2 \\
\hline \multirow[t]{2}{*}{ LDTg-Ch6 } & $J$ & 1 & 1 & 0 \\
\hline & $\mathrm{K}$ & 1 & 1 & 0 \\
\hline
\end{tabular}

The injection sites were divided into four groups: injections largely confined to the PPN-Ch 5, this group was further subdivided into injections which were centered on PPN-Ch 5 and injections which involved the lateral half of PPNCh 5; injections largely confined to the MEA; injections involving the PPNCh 5 and the MEA (PPN-Ch5 + MEA); injections confined to the LDTg-Ch 6. The location of the injection site is denoted by a letter which corresponds to the schematic representation of the injection site in Figure 2. The density of anterograde labeling was assessed in three representative sections from the caudal, middle, and rostral thirds of the ipsilateral STN of each animal and is indicated by the following key: $0=$ no labeling; 1 = sparse labeling; $2=$ moderate labeling; 3 = dense labeling.

(0.984 $\pm 0.38, n=79)$. The majority of terminals forming asymmetrical synaptic contacts were immunonegative for GABA (index of GABA immunoreactivity, $2.233 \pm 0.253, n=$ 36) (Figs. 5, 6B), although a minority were immunopositive for GABA $(21.37 \pm 3.854, n=5$ ) (Figs. 5, 6A). All terminals forming symmetrical synaptic contacts were immunopositive for GABA $(23.229 \pm 1.164, n=22)$ (Figs. 5, 6A, 9, 10). The GABA-immunopositive boutons that formed asymmetric and symmetric synaptic contacts were not significantly different in their immunoreactivity for glutamate $(1.028 \pm 0.202, n=5$ and $0.984 \pm 0.38, n=79$, respectively).

(2) Boutons anterogradely labeled from the MTg. The amino acid immunoreactivity of anterogradely labeled presynaptic and synaptic boutons (all of which formed asymmetric synaptic contacts) was assessed from similar numbers of boutons that werc derived from three animals that received injections in the parts of the MTg that projected most heavily to the STN, that is, the PPN-Ch 5 (Fig. $2 B, n=19$ ) or the MEA (Fig. $2 G, n=21$ ) or both regions (Fig. $2 H, n=16$ ). The boutons anterogradely labeled from the MTg could be divided into two groups on the basis of their immunoreactivity for GABA and glutamate. The GABA-immunopositive boutons (17.205 $\perp 1.276, n=17)$ had significantly higher $(p<0.001)$ immunoreactivity for GABA
(Figs. 5, 6) than the immunonegative boutons $(2.348 \pm 0.258$, $n=39$ ) (Figs. 5,8 ). The glutamate immunoreactivity of the anterogradely labeled GABA-immunonegative boutons (1.357 $\pm 0.115, n=39$ ) was significantly higher (Mann-Whitney $U$ test, $p<0.006$ ) than that found in nonanterogradely labeled terminals that formed symmetrical synaptic contacts (see above for values) (Fig. 7, 8). In contrast, the glutamate immunoreactivity of the anterogradely labeled GABA-immunopositive boutons $(0.959 \pm 0.121, n=17)$ was not significantly different to that found in terminals that formed symmetrical synaptic contacts (Figs 6, 7).

(3) ChAT-immunopositive boutons. The amino acid content of ChAT-immunopositive boutons in the STN was addressed by pre-embedding immunocytochemistry for ChAT and post-embedding immunocytochemistry for GABA and glutamate. Since immunocytochemistry for ChAT is more successful with fixatives containing a low glutaraldehyde concentration (less than $0.5 \%$ ) and immunocytochemistry for amino acids is successful with a relatively high concentration of glutaraldehyde in the perfusion fixative (greater than $0.5 \%$ ), animals for the combined detection of ChAT and amino acids were perfused with fixatives containing $0.3-0.5 \%$ glutaraldehyde. Using this 'compromise' fixative ChAT and GABA immunolabeling were detected in two out of four animals. Glutamate immunocytochemistry was not successful in any of the four animals. The pattern of immunolabeling for GABA was assessed quantitatively as described above. Since the fixation conditions were different, the data were not pooled with the data obtained from the other post-embedding experiments. The pattern of GABA immunolabeling in the subthalamic nucleus was very similar to that observed in tissue which had been perfused with fixatives that were optimal for the detection of amino acids. The index of GABA immunoreactivity in terminals forming symmetrical synaptic contacts was $12.108 \pm 0.767(n=34)$ and was significantly greater $(p<$ 0.001 , Mann-Whitney $U$ test) than that found in ChAT-immunopositive terminals ( $2.177 \pm 0.33, n=35$; Fig. 9) or nonanterogradely labeled terminals that formed asymmetrical synapses $(1.033 \pm 0.171, n=31)$ which were thus defined as GABA immunonegative.

\section{Convergence of anterogradely labeled terminals or ChAT- immunopositive terminals and GABAergic terminals onto individual neurons in the subthalamic nucleus}

The analysis of anterogradely labeled terminals in sections that were immunolabeled by the post-embedding method for GABA revealed that the terminals derived from the $\mathrm{MTg}$ made convergent synaptic contact with dendrites that receive input from GABA-immunopositive terminals (Fig. 10, $n=10$ from three cases illustrated Fig. $2 B, G, H$ ). On each occasion the anterogradely labeled terminal was itself GABA-negative and the GABApositive terminal displayed characteristics typical of those derived from the globus pallidus, that is, they were large boutons, contained several mitochondria, relatively few vesicles that congregated at the active zone and formed symmetrical synaptic contacts. Convergence of a similar nature was observed between

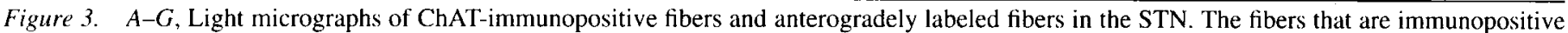

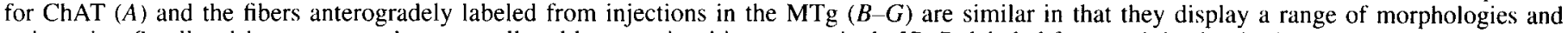

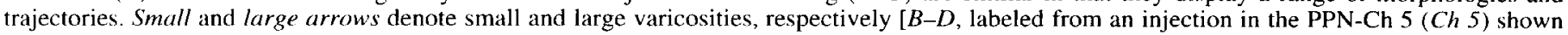

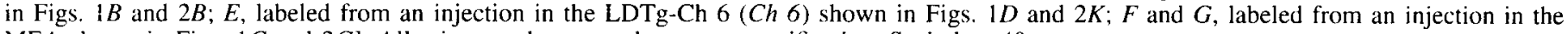
MEA shown in Figs. $1 C$ and $2 G$ ]. All micrographs are at the same magnification. Scale bar, $40 \mu \mathrm{m}$. 

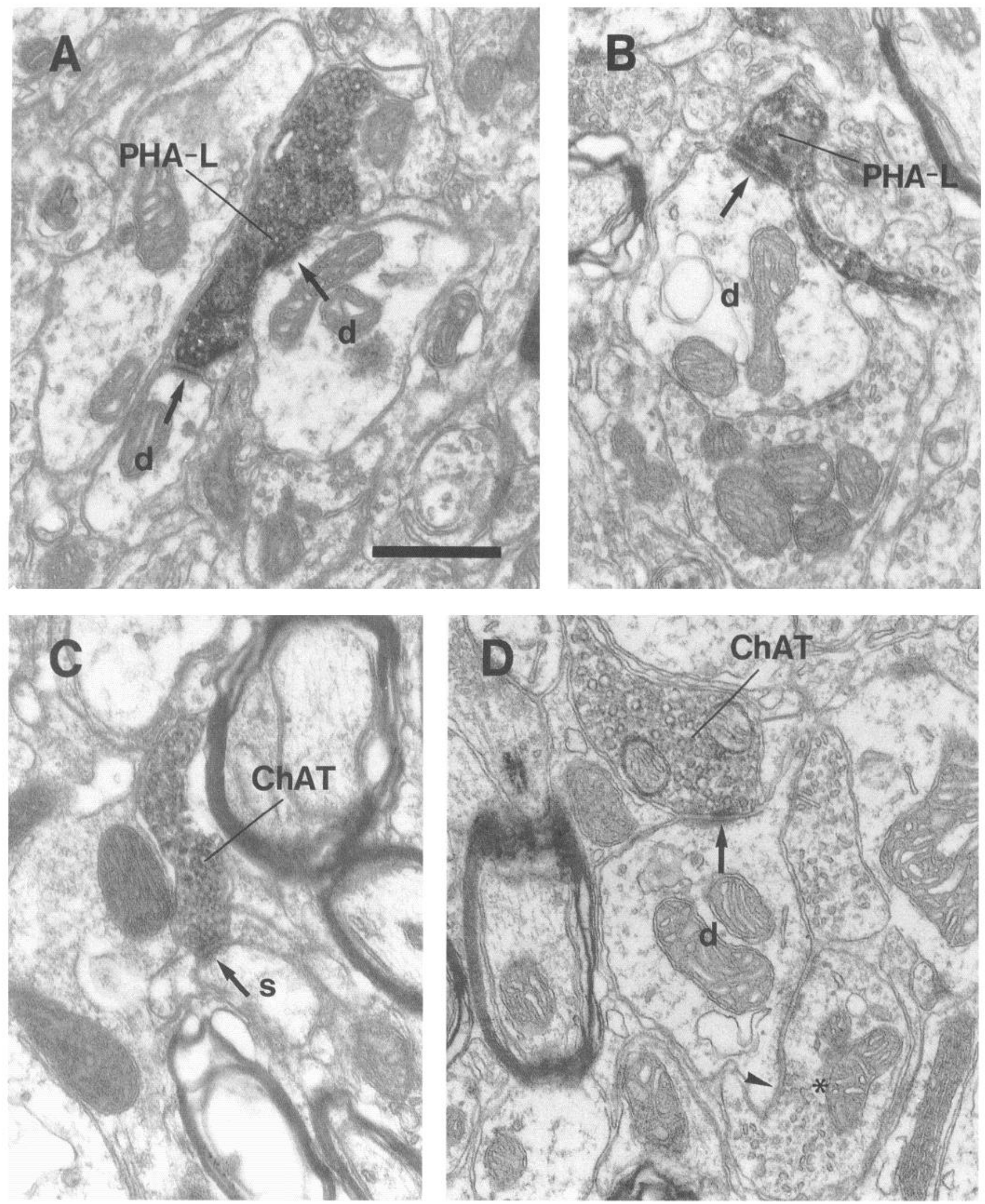

Figure 4. $A-D$, Electron micrographs demonstrating the morphology of synaptic terminals anterogradely labeled from a large deposit of PHA-L ( $A, B$ ) in the MTg (shown in Figs. $1 A$ and $2 L$ ) and immunocytochemically labeled for ChAT $(C, D)$ in the STN. The terminals were revealed immunocytochemically using DAB as the chromogen; the electron dense reaction product fills the synaptic terminals and adheres to their intracellular organelles. Both the anterogradely labeled terminals $(A, B)$ and the ChAT-immunopositive terminals $(C, D)$ form asymmetric synaptic contacts (arrows) with small diameter dendritic shafts $(d)$ or spines $(s)$ of subthalamic neurons. Note in $D$ that one of the dendrites postsynaptic to a ChAT-immunopositive terminal also receives symmetrical synaptic input (arrowhead) from an unlabeled terminal (asterisk). All micrographs are at the same magnification. Scale bar, $0.5 \mu \mathrm{m}$. 
GABA immunoreactivity of boutons and synaptic terminals in the STN

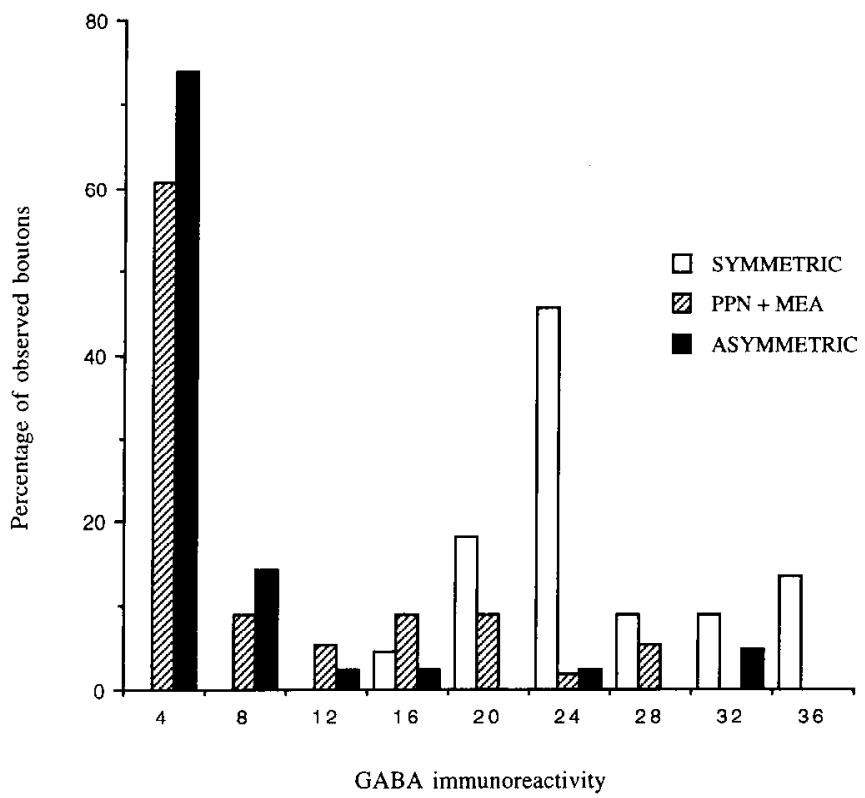

Figure 5. Frequency distribution of the level of GABA immunoreactivity associated with axonal boutons and synaptic terminals in the STN GABA immunoreactivity is represented as the ratio of the density of immunogold particles overlying the bouton to that overlying dendrites and cell bodies of subthalamic neurons in the same section. SYMMETRIC, Terminals forming symmetrical synapses; ASYMMETRIC, terminals forming asymmetrical synapses; $P P N+M E A$, boutons anterogradely from deposits of BDA in the MTg.

terminals that displayed immunoreactivity for ChAT and GABA-positive terminals. (Fig. 9, $n=7,2$ cases). Convergence was not observed between terminals anterogradely labeled from the MTg that were GABA-immunopositive and GABA-immunopositive terminals that displayed the features that were typical of pallidal terminals.

\section{Discussion}

The major findings of this study provide new insights into the origin, ncurochemistry, and synaptic organization of the mesopontine tegmental projection to the STN and demonstrate that the system is more complex than previously thought. The major projections to the STN are predominantly ipsilateral and arise from PPN-Ch 5, the adjacent and largely noncholinergic MEA and to a smaller extent, the LDTg nucleus. The fact that part of this projection arises in the region of the cholinergic neurons in the MTg and the similarity between the morphology, trajectory, and synaptology of the anterogradely labeled fibers and ChATimmunopositive fibers, suggests that at least one component of the projection is cholinergic. Furthermore, based on the postembedding immunocytochemical data, it is likely that additional components of the projection use GABA as a neurotransmitter and some of the projecting neurons use glutamate as their neurotransmitter. Finally, individual subthalamic neurons receive convergent synaptic input from terminals anterogradely labeled from the $\mathrm{MTg}$ or cholinergic terminals together with $\mathrm{G} \Lambda \mathrm{B} \Lambda$ immunoreactive terminals that form symmetrical synaptic contacts and are probably derived from the globus pallidus. Taken together these findings imply that the MTg sends cholinergic, GABAergic and glutamatergic projections to the STN where at least one of the roles is to modulate the indirect pathway of information-flow through the basal ganglia that is carried in the pallidosubthalamic projection.

The site of origin of the mesopontine tegmental projection to the subthalamic nucleus

One of the major objectives of the present study was to clarify the exact origin of the projections from the MTg to the STN. This was achieved by the use of small deposits of anterograde tracers and by exactly delineating these deposits in relation to the known chemoarchitecture of the region. Thus, the sections were histochemically counterstained to reveal NADPH diaphorase which in this region is a faithful marker of cholinergic neurons (Vincent et al., 1983). We were thus able to clarify the conflicting reports in the literature by demonstrating that at least three distinct subregions of the MTg innervate the STN, to varying degrees. First, the area defined as the MEA (Rye et al., 1987; Lee et al., 1988) which consists largely of noncholinergic neurons, provides a major innervation of the STN. Secondly, de posits of tracers that were confined to, or largely included, the diaphorase-positive (i.e., cholinergic) neurons which are more laterally placed, demonstrate that the region we have defined as PPN-Ch5 also gives rise to a major projection to the subthalamic nucleus (Woolf and Butcher, 1986; Spann and Grofova, 1989). Thirdly, the deposits of tracers that included the cholinergic neurons of the laterodorsal tegmental nucleus, demonstrate that this region also innervates the subthalamic nucleus albeit to a smaller extent (Woolf and Butcher, 1986). It is thus clear that the mesopontine projection is more complex than previously thought and does not arise exclusively, or indeed predominantly, from one or other subregion of the MTg or a neurochemically specific subgroup of the neurons in the mesopontine tegmentum (Woolf and Butcher, 1986; Lee et al., 1988; Spann and Grofova, 1989).

\section{Neurochemistry of the mesopontine tegmental projection to the STN}

In addition to demonstrating that the projection from the $\mathrm{MTg}$ to the STN is heterogeneous with respect to its origin, the present data demonstrate that the projection is also heterogeneous with respect to the neurochemistry of its synaptic terminals. At least two, and possibly three or more, neurochemically distinct subpopulations of anterogradely labeled terminals were identified in the STN. On the basis of (1) the location of the tracer deposits in relation to identified cholinergic neurons, (2) the similarity in the morphology and synaptology of the anterogradely labeled terminals and ChAT-immunopositive terminals, and (3) previous combined retrograde labeling and immunocytochemical studies (Woolf and Butcher, 1986; Lee et al., 1988), it is likely that a component of the projection arises from cholinergic neurons of the MTg. A cholinergic input to the STN is also compatible with the presence of immunoreactivity for $m 3$ receptors in subthalamic neurons (Levey et al., 1994) and the demonstration of a dense network of ChAT-immunoreactive fibers in the nucleus (Mesulam et al., 1992; present study). On the basis of the different densities of anterograde labeling that we observed and the numbers of cholinergic neurons that were retrogradely labeled in the pedunculopontine and laterodorsal tegmental nuclei (Woolf and Butcher, 1986), the major part of the cholinergic projection is likely to arise from the cholinergic neurons of the pedunculopontine nucleus and only a minor part from the laterodorsal tegemental nucleus. It must remembered, however, that the region we defined as the PPN-Ch 5 also contains noncholi- 

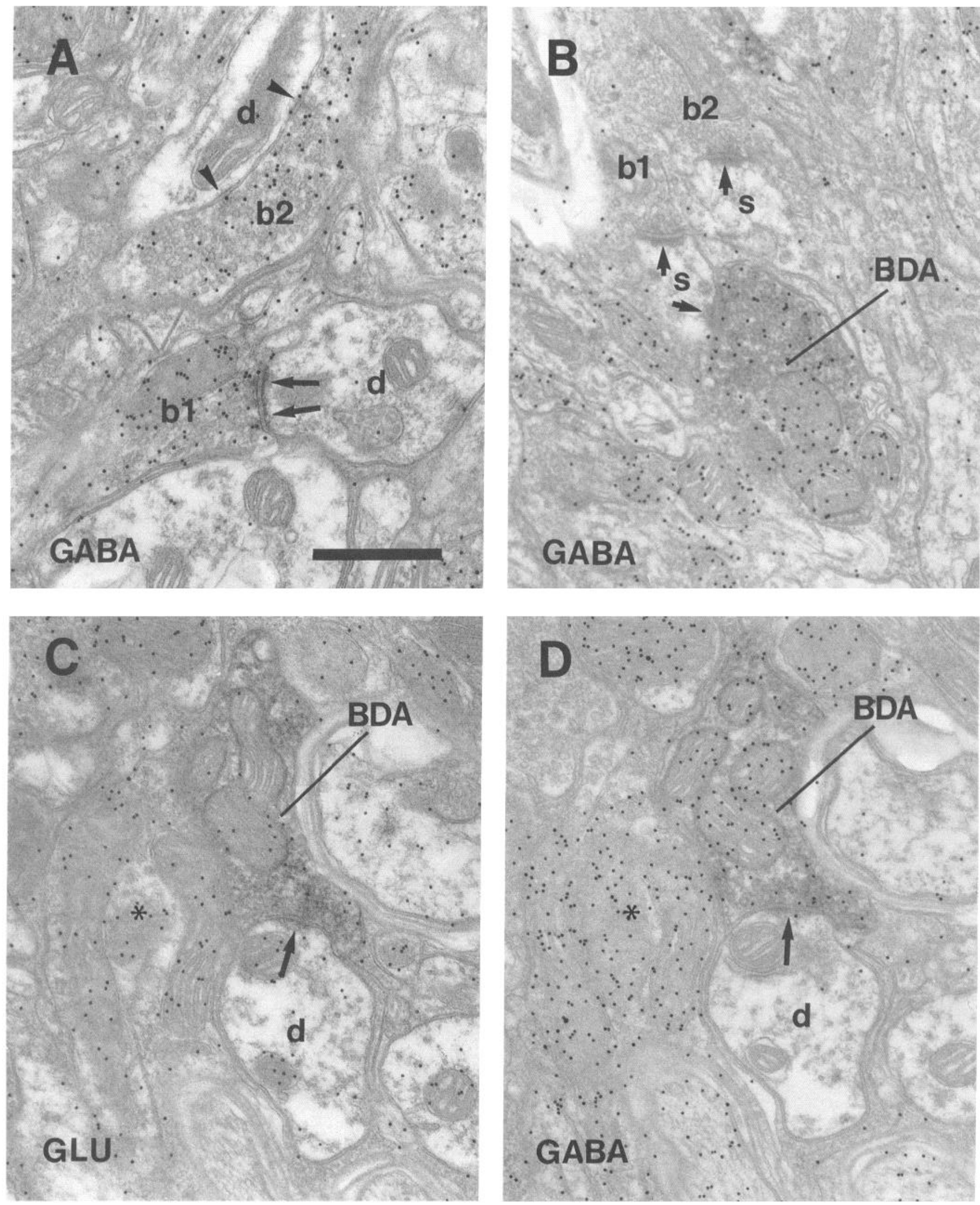

Figure 6. Electron micrographs of ultrathin sections of the STN that have been immunolabeled for GABA $(A, B, D)$ and glutamate $(G L U, C)$. $A$, Two GABA-immunopositive boutons (index of GABA immunoreactivity $=31.19$ and 23.29 for b1 and b2, respectively) form asymmetrical (arrows) and symmetrical contact (arrowheads) with dendrites $(d)$ of subthalamic neurons, respectively. B, A GABA-immunopositive bouton (index of GABA immunoreactivity $=25.86$ ) anterogradely labeled from an injection of BDA in the MTg makes asymmetrical synaptic contact (arrow) with a spine $(s)$ of a subthalamic neuron. This spine and an adjacent spine $(s)$ also receive asymmetrical synaptic contact (arrows) from two GABAimmunonegative terminals $(\mathrm{b} 1=1.18$, and $\mathrm{b} 2=0.81)$. $C$ and $D$, Adjacent ultrathin sections that have been immunolabeled for glutamate $(C$, 
Glutamate immunoreactivity of boutons and synaptic terminals in the STN

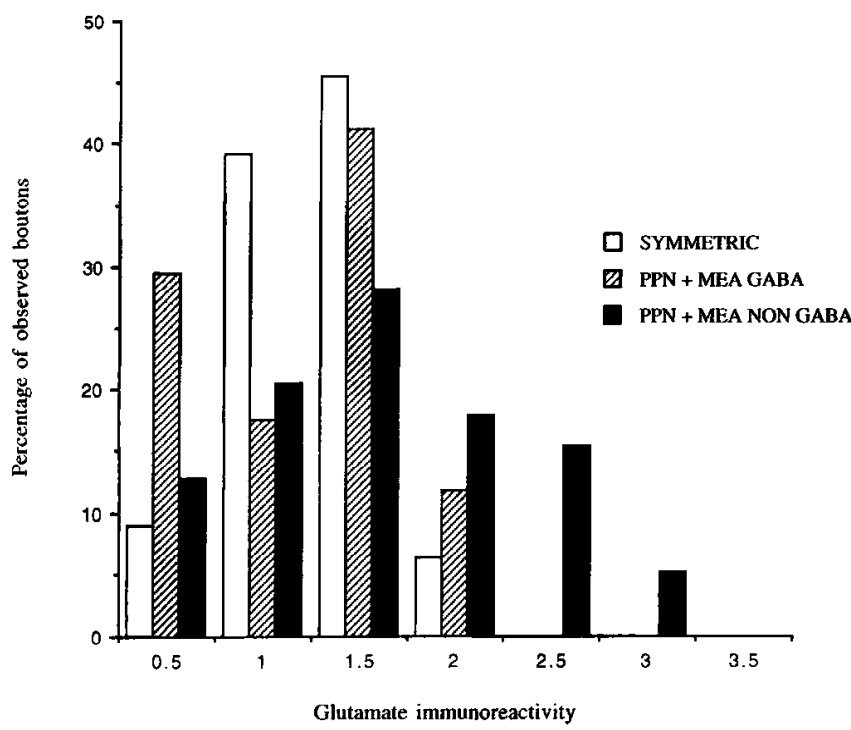

Figure 7. Frequency distribution of the level of glutamate immunoreactivity associated with boutons and synaptic terminals in the STN that were anterogradely labeled from the MTg. Glutamate immunoreactivity is represented as the ratio of the density of immunogold particle overlying the bouton to that overlying terminals forming symmetrical synapses in the same section. SYMMETRIC, Terminals forming symmetrical synapses; $P P N+M E A G A B A$, boutons and synaptic terminals that were anterogradely labeled from deposits in the $M T g$ and were immunopositive for GABA; $P P N+M E A N O N G A B A$, boutons and synaptic terminals that were anterogradely labeled from deposits in the $\mathrm{MTg}$ and were immunonegative for GABA. The anterogradely labeled GABA-immunonegative boutons and synaptic terminals are significantly enriched in glutamate compared to that found in the anterogradely labeled GABA-immunopositive boutons and synaptic tcrminals.

nergic neurons (Clements and Grant, 1990; Jones, 1990, 1993; Lavoie and Parent, 1994b,c) and the present anterograde tracing does not distinguish which neurons gave rise to the anterograde labeling in the STN. A cholinergic innervation of the substantia nigra has also been reported (Bolam et al., 1991; Mesulam et al., 1992)

The post-embedding immunocytochemistry for glutamate and GABA combined with the anterograde labeling revealed that components of the projection contain relatively high levels of GABA or glutamate and therefore presumably use them as a neurotransmitter. At least two types of anterogradely labeled terminals with respect to their amino acid content were identified, all of which formed asymmetrical synaptic specializations. One population was significantly enriched in glutamate immunoreactivity and was GABA-immunonegative and is therefore likely to be glutamatergic (Somogyi et al., 1986; Ottersen, 1989; Ottersen and Storm-Mathisen, 1989; Llewellyn-Smith, 1992; Phend et al., 1992; Rinvik and Ottersen, 1993; Kharazia and Weinberg, 1994; Valtschanoff et al., 1994; Bevan et al., 1995). This observation is supported by the reports of high levels of glutamate immunoreactivity in neurons in the regions that were injected in this study (Clements and Grant, 1990; Lavoie and Parent, 1994b,c). The level of glutamate enrichment in this "population" of boutons was however, highly variable. Indeed, the levels of glutamate were in general more variable and lower than that found in anterogradely labeled cortical and thalamic boutons in the STN (Bevan et al., 1995). This variability may be a reflection of the heterogeneities in glutamate immunoreactivity that have been observed in neurons in the regions of the MTg that were injected in this study (Clements and Grant, 1990; Lavoie and Parent, 1994b,c), rather than a technical artefact, since the same approach was used to study the glutamate immunoreactivity of cortical and thalamic terminals in the STN (Bevan et al., 1995). A glutamatergic component to the projection from the MTg to the substantia nigra has also been demonstrated on anatomical and electrophysiological grounds (Scarnatti et al., 1987; Lavoie and Parent 1994b,c; Smith et al., 1994b). A second population of terminals was significantly enriched in GABA-immunorcactivity but poor in glutamate and are therefore presumably GABAergic structures (Hodgson et al., 1985; Somogyi and Hodgson, 1985; Somogyi et al., 1985, 1986; Ottersen, 1989). This population of GABAergic synaptic terminals was unusual in that they formed asymmetrical synaptic contacts, that is, the type of specialization not usually associated with GABAergic terminals. However, populations of GABAergic (GAD-positive) terminals forming asymmetrical synapses have also been observed in other brain regions including the substantia nigra (Ribak et al., 1976; Nitsch and Riesenberg, 1988; Bolam and Smith, 1990). A GABAergic component of the mesopontine tegmental projection to the STN is consistent with the presence GABAergic neurons in the areas in which anterograde tracers were injected (Jones, 1990 , 1993). Similarly a small proportion of terminals in the primate substantia nigra that were anterogradely labeled from the MTg have been demonstrated to display GABA immunoreactivity (Smith et al., 1994b)

An important issue that arises is the relationship between the imunoreactivity for amino acids and the cholinergic terminals, since some of the cholinergic neurons in the pedunculopontine region have been shown to be strongly glutamate-immunoreactive (Clements and Grant, 1990; Lavoie and Parent, 1994b.c). In the present study we were unable to examine the levels of glutamate-immunoreactivity in the ChAT-immunoreactive terminals since the recognition of the two antigens by immunocytochemistry required different fixatives (see also Ottersen, 1989). However, it was possible to detect both ChAT- and GABA-immunoreactivity in the same tissue and from these experiments it was clear that the cholinergic terminals display relatively very low GABA-immunoreactivity, an observation that is consistent with the failure to detect GABA immunoreactivity in cholinergic neurons in the MTg (Jones, 1990, 1993).

\section{Synaptology of the mesopontine tegmental projection to the STN}

The gross and ultrastructural morphologies of the fibers anterogradely labeled from injections of BDA confined either to the PPN-Ch 5 or the MEA were not clearly related to the site of injection. Thus injections in both sites gave rise to fibers and varicosities of variable size and trajectory and synaptic terminals

$\leftarrow$

GLU) and GABA $(D)$. A bouton anterogradely labeled with BDA from an injection in the MTg makes asymmetrical synaptic contact (arrow) with a dendrite $(d)$ of a subthalamic neuron. The bouton is GABA-immunopositive (19.92) but has a relatively low index of glutamate immunoreactivity (1.36). Another GABA-immunopositive bouton (asterisk; 31.49) lies adjacent to the BDA-labeled bouton. All micrographs are at the same magnification. Scale bar, $0.5 \mu \mathrm{m}$. 

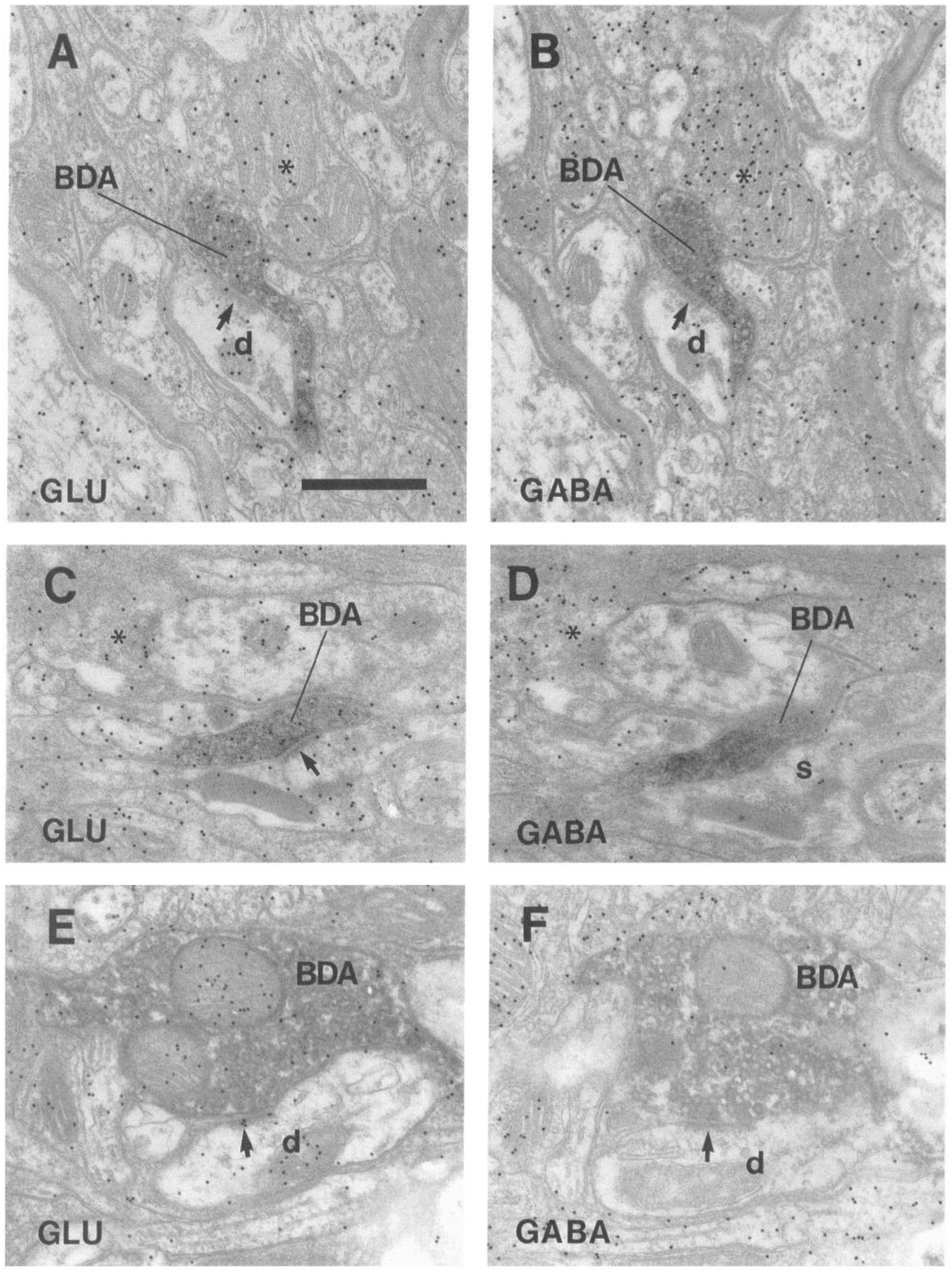

Figure 8. Pairs of electron micrographs of serial sections of synaptic terminals in the STN that were anterogradely labeled from BDA injections in the MTg. One of each pair has been immunolabeled for glutamate $(A, C, E ; G L U)$ and the other for GABA $(B, D, F)$. The synaptic terminals are associated with relatively high levels of glutamate immunoreactivity $(A=2.07, C=1.60, E=2.05)$ and are GABA immunonegative $(B=$ 

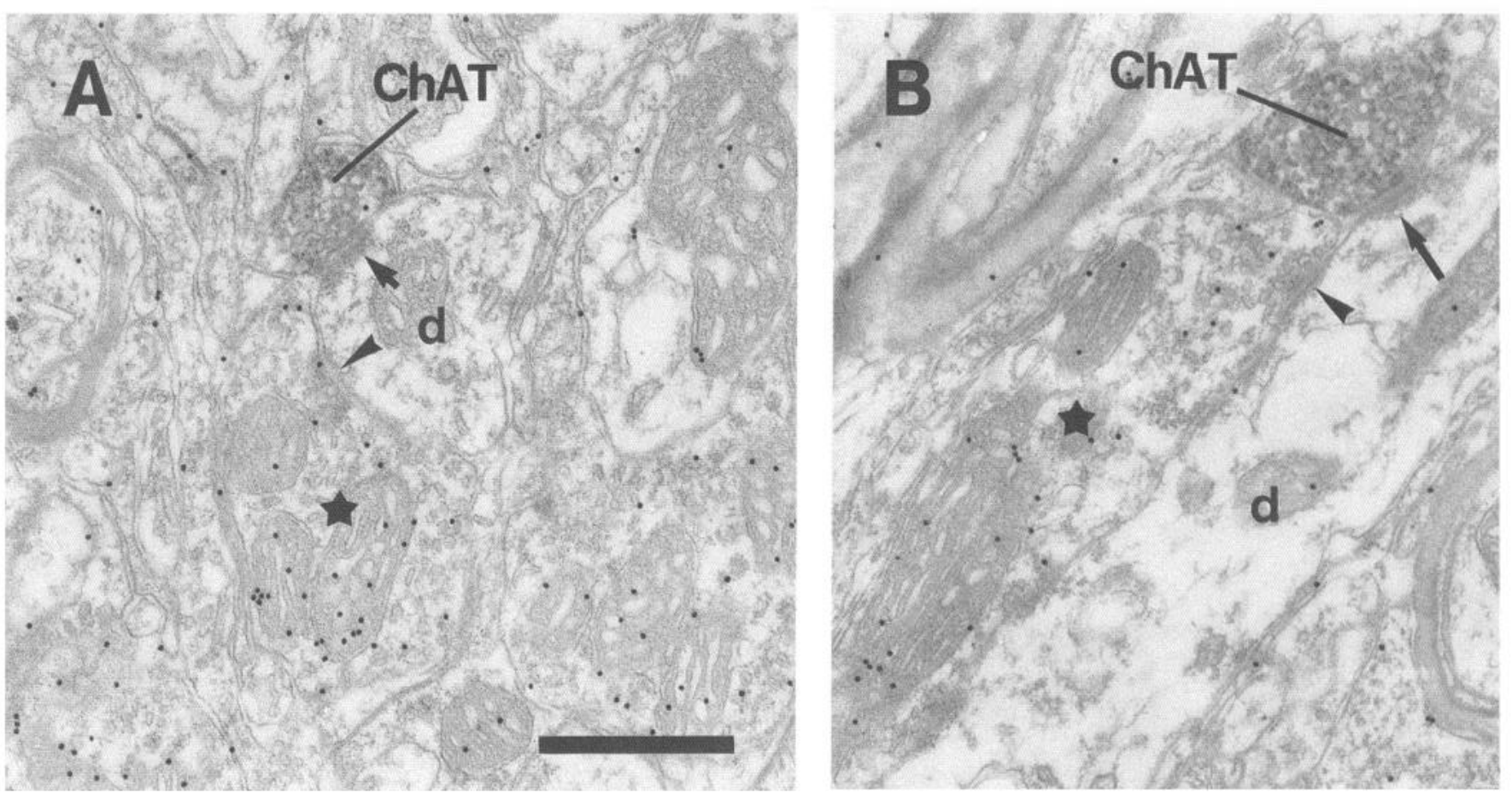

Figure 9. $A$ and $B$, Electron micrographs of ChAT-immunopositive and GABA-immunopositive terminals in convergent synaptic contact with dendrites of individual subthalamic neurons. The ChAT-immunopositive boutons contain relatively low GABA-immunoreactivity $(A=4.03 ; B=$ 0 ) and form asymmetrical synaptic contacts (arrows) with small diameter dendrites (d) of subthalamic neurons. The same dendrites are also postsynaptic to terminals that form symmetrical synaptic contacts (arrowheads) and are GABA-immunopositive $($ stars; $A=17.21 ; B=7.02)$. These boutons are probably derived from the globus pallidus. All micrographs are at the same magnification. Scale bar, $0.5 \mu \mathrm{m}$.

of variable size. The consistent feature was that all terminals anterogradely labeled from the mesopontine tegmental region formed asymmetrical synaptic contacts with the dendrites and spines of subthalamic neurons. This finding is in contrast to Sugimoto and Hattori (1984) who reported that some of the terminals anterogradely labeled from this region form symmetrical synaptic contacts. One explanation for this discrepancy is that we observed that some of the anterogradely terminals formed very short asymmetrical synaptic contacts, that were asymmetric in only two or three ultrathin sections and in the ultrathin sections adjacent to these, the synapses appeared symmetrical. Only by following these terminals throughout their synapses, were we able to determine their true symmetry.

The present study demonstrates that ChAT-immunopositive terminals form asymmetrical synaptic contacts with the dendritic shafts and spines of subthalamic neurons. Indeed the gross and ultrastructural morphology of ChAT-immunopositive fibers and terminals was similar to terminals anterogradely labeled from the MTg. However ChAT-immunopositive synaptic terminals were significantly smaller than the population of terminals anterogradely labeled with PHA-L. The reason for this may be that anterograde tracing labeled cholinergic, glutamatergic and GABAergic terminals whereas immunocytochemistry for ChAT only labeled cholinergic terminals (some of which may also have been glutamatergic).

\section{Functional considerations}

Terminals anterogradely labeled from the MTg that were GABA-immunonegative and cholinergic terminals made convergent synaptic contact with subthalamic neurons that received symmetrical synaptic input from GABA-immunopositive terminals that were presumably derived from the globus pallidus. These findings are consistent with the findings of an earlier study in which degenerating terminals (following lesion of the MTg that included the area studied here) were in synaptic contact with subthalamic neurons that also received synaptic input from terminals anterogradely labeled from the globus pallidus in cats (Moriizumi et al., 1987). Taken together, these findings indicate that cholinergic and/or possibly glutamatergic components of the mesopontine tegmental projection to the STN act on the same neurons that receive input from the globus pallidus. The pallidal projection to the STN forms part of a so-called "indirect pathway" of information flow through the basal ganglia (cortex to neostriatum to globus pallidus to STN to basal ganglia output nuclei) (Albin et al., 1989; Alexander et al., 1990; Bevan et al., 1994a,b). The physiological and pathophysiological action of this pathway is associated with the increased inhibition of basal ganglia targets and thus the suppression of movement (Crossman, 1987; Albin et al., 1989; Chevalier and Deniau, 1990; DeLong, 1990; Fujimoto and Kita, 1992; Matsumara et al.,

$\leftarrow$

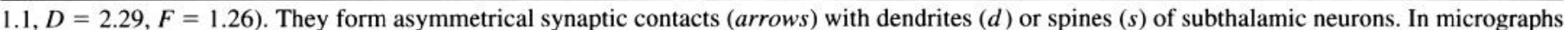

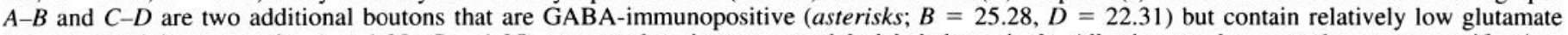

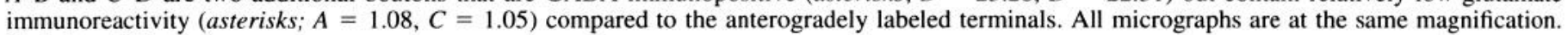
Scale bar, $0.5 \mu \mathrm{m}$. 

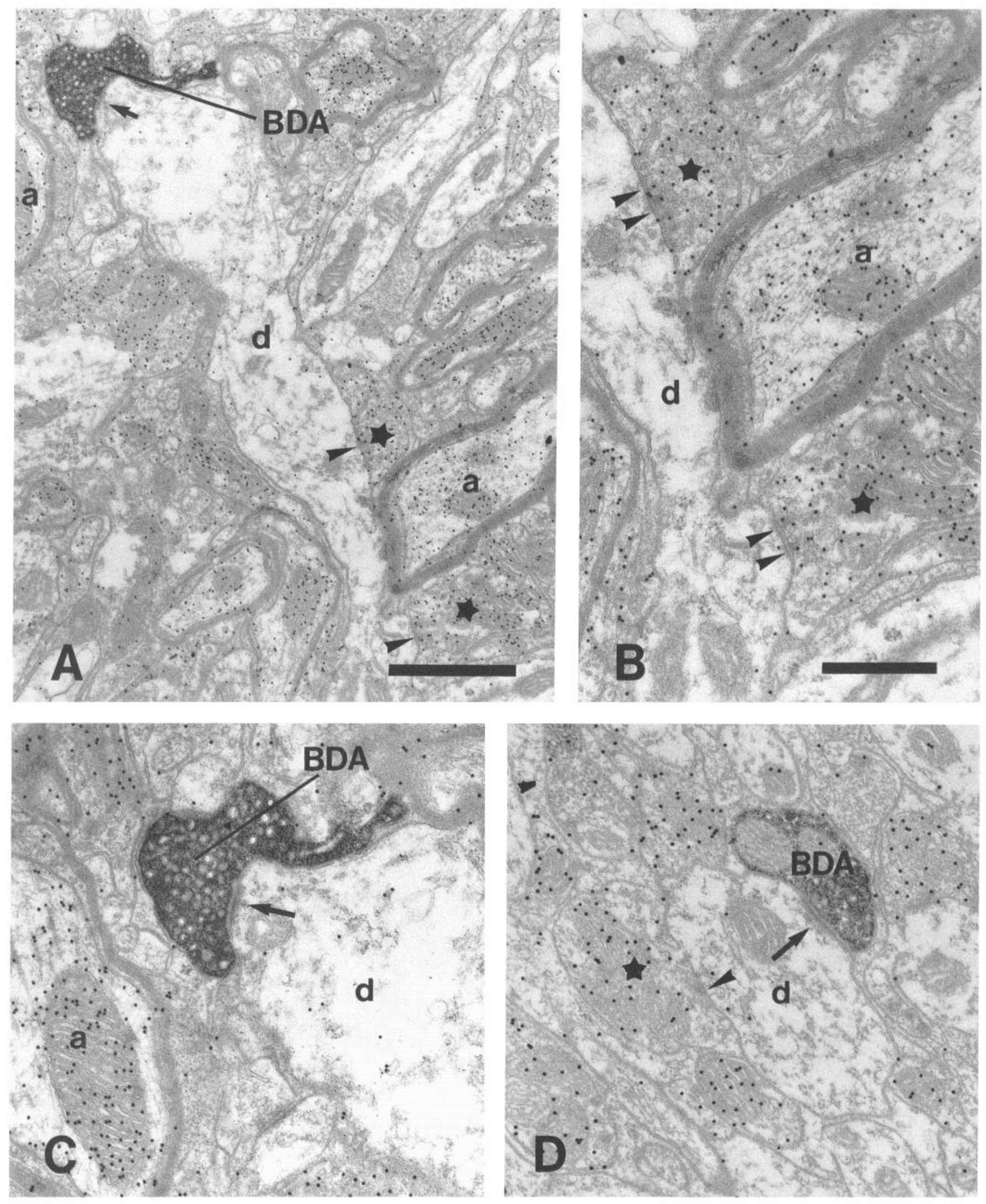

Figure 10. $A-D$, Electron micrographs of terminals anterogradely labeled from injections of BDA in MTg and GABA-immunopositive terminals in convergent synaptic contact with dendrites of individual subthalamic neurons. The anterogradely labeled boutons are GABA-immunonegative $(C=0.96 ; D=1.98)$ and form asymmetrical synaptic contacts (arrows) with dendrites $(d)$ of subthalamic neurons that also receive symmetrical synaptic input (arrowheads) from GABA-immunopositive terminals (stars; upper terminal in $B=21.41 ;$ lower terminal in $B=18.31 ; D=16.2$ ). These terminals are probably derived from the globus pallidus. Scale bars: $A, 1 \mu \mathrm{m} ; B, 0.5 \mu \mathrm{m}$ (also applies to $C$ and $D$ ). 
1992; Bergman, 1994; Wichmann et al., 1994a,b) and acts to oppose the action of the direct pathway (cortex-neostriatumbasal ganglia output nuclei), which is associated with movement (Hikosaka and Wurtz, 1985a,b; Albin et al., 1989; Chevalier and Deniau, 1990; DeLong, 1990; Fujimoto and Kita, 1992). Our findings demonstrate the cholinergic and possibly the glutamatergic components of the mesopontine tegmental projection are in a position to modulate the flow of information in this indirect pathway at the level of the STN. Thus the projection from the MTg to the STN represents a key interface betwecn brainstem systems involved in locomotion, sleep-wake cycles and arousal and basal ganglia systems that are critical to movement. The complexity of this interface is highlighted by our demonstration of cholinergic, glutamatergic, and GABAergic components to this projection. A thorough knowledge of the input-output organization of the MTg with respect to its projections to STN is necessary to further our understanding of the functional role of this projection. The demonstration of a dense projection from the cerebellum to the areas of MTg (Hazrati and Parent, 1992) that project to the STN (Lavoie and Parent 1994a) suggests that this may be one route through which the cerebellum influences the basal ganglia.

\section{References}

Abdullah LH, Ordronneau P, Petrusz P (1992) Molecular requirements for hapten binding to antibodies against glutamate and aspartate. Neuroscience 51:729-738.

Albin RL, Young AB, Penney JB (1989) The functional anatomy of basal ganglia disorders. Trends Neurosci 12:366-375.

Alexander GE, Crutcher MD (1990) Functional architecture of basal ganglia circuits: neural substrates of parallel processing. Trends Neurosci 13:266-271.

Aziz TZ, Peggs D, Sambrook MA, Crossman AR (1991) Lesion of the subthalamic nucleus for the alleviation of 1 -methyl-4-phenyl-1,2,3,6,terahydropyridine (MPTP)-induced parkinsonism in the primate. Mov Disord 6:288-292.

Benazzouz A, Gross C, Féger J, Boraud T, Bioulac B. (1993) Reversal of rigidity and improvement in motor performance by subthalamic high-frequency stimulation in MPTP-treated monkeys. Eur $\mathbf{J}$ Neurosci 5:382-389.

Bergman H, Wichmann T, DeLong MR (1990) Reversal of experimental parkinsonism by lesions of the subthalamic nucleus. Science 249 : 1436-1438.

Bergman H, Wichmann T, Karmon B, DeLong MR (1994) The primate subthalamic nucleus. II. Neuronal activity in the MPTP model of parkinsonism. J Neurophysiol 72:521-530.

Bevan MD, Bolam JP, Crossman AR (1994a) Convergent synaptic input from the neostriatum and subthalamus onto identified nigrothalamic neurons in the rat. Eur J Neurosci 6:320-334.

Bevan MD, Crossman AR, Bolam JP (1994b) Neurons projecting from the entopeduncular nucleus to the thalamus receive convergent synaptic inputs from the subthalamic nucleus and the neostriatum in the rat. Brain Res 659:99-109.

Bevan MD, Francis CM, Bolam JP (1995) The glutamate-enriched cortical and thalamic input to neurons in the subthalamic nucleus of the rat: convergence with GABA-positive terminals. J Comp Neurol, in press.

Bolam JP, Smith Y (1990) The GABA and substance P input to dopaminergic neurons in the substantia nigra of the rat. Brain Res 529: $57-78$.

Bolam JP, Francis CM, Henderson Z (1991) Cholinergic inputs to dopaminergic neurons in the substantia nigra. A double immunocytochemical study. Neuroscience 41:483-494.

Bowsher D, (1975) Diencephalic projections from the midbrain reticular formation. Brain Res 95:211-220.

Brotchie JM, Mitchell IJ, Sambrook MA, Crossman AR (1991) Alleviation of parkinsonism by antagonism of EAA transmission in the medial segment of the globus pallidus in rat and primate. Mov Disord 6:133-138.

Butcher LL, Semba K (1989) Reassessing the cholinergic basal fore- brain: nomenclature schemata and concepts. Trends Ncurosci 12:48.3485.

Canteras RD, Shammah-Lagnado SJ, Silva BA, Ricardo JA (1990) Af ferent connections of the subthalamic nucleus: a combined retrograde and anterograde horseradish peroxidase study in the rat. Brain Res 513:43-59.

Chevalier G, Deniau JM (1990) Disinhibition as a basic process in the expression of striatal functions. Trends Neurosci 13:277-280.

Clements JR, Grant S (1990) Glutamate-like immunoreactivity in neurons of the laterodorsal tegmental and pedunculopontine nuclei in the rat. Neurosci Lett 120:70-73.

Crossman AR (1987) Primate models of dyskinesia: an experimental approach to the study of basal ganglia-related involuntary movement disorders. Neuroscience 21:1-40.

Delong MR (1990) Primate models of movement disorders of basal ganglia origin. Trends Neurosci 13:281-285.

Filion M, Tremblay L (1991) Abnormal spontaneous activity of globus pallidus neurons in monkeys with MPTP-induced parkinsonism. Brain Res 547:142-151.

Fujimoto K, Kita H. (1992) Responses of rat substantia nigra pars reticulata units to cortical stimulation. Neurosci Lett 142:105-109.

Garcia-Rill E (1991) The pedunculopontine nucleus. Prog Neurobiol $36: 363-389$.

Gerfen CR, Sawchenko PE (1984) An anterograde neuroanatomical tracing method that shows the detailed morphology of neurons, their axons and terminals: immunohistochemical localization of an axonally transported plant lectin Phaseolus vulgaris-leucoagglutinin (PHA-L). Brain Res 290:219-238.

Groenewegen HJ, Berendse HW (1990) Connections of the subthalamic nucleus with ventral striatopallidal parts of the basal ganglia in the rat. J Comp Neurol 294:607-622.

Groenewegen HJ Berendse HW, Haber SN (1993) Organization of the output of the ventral striatopallidal system in the rat: ventral pallidal efferents. Neuroscience 57:113-142.

Gonya-Magee TA (1983) An electrophysiological characterization of projections from the pedunculopontine area to entopeduncular nucleus and globus pallidus in the cat. Exp Brain Res 49:611 624.

Graybiel AM (1977) Direct and indirect preoculomotor pathways of the brainstem: an autoradiographic study of the pontine reticular formation in the cat. $J$ Comp Neurol 175:37-78.

Hazrati LN, Parent A (1992) Projection from the deep cerebellar nuclei to the pedunculopontine nucleus in the squirrel monkey. Brain Res 585:267-271.

Hepler JR, Toomin CS, McCarthy KD, Conti F, Battaglia G, Rustioni A. Petrusz P (1988) Characterization of antisera to glutamate and aspartate. J Histochem Cytochem 36:13-22.

Hikosaka O, Wurtz RH (1985a) Modification of saccadic eye movements by GABA-related substances. 1. Effects of muscimol and bicuculline in the monkey superior colliculus. J Neurophysiol 53:266291.

Hikosaka O, Wurtz RH (1985b) Modification of saccadic eye movements by GABA-related substances. 2. Effects of muscimol in the monkey substantia nigra pars reticulata. J Neurophysiol 53:292-308.

Hodgson AJ, Penke B, Erdei A, Chubb IW, Somogyi P (1985) Antisera to $\gamma$-aminobutyric acid. I. Production and characterisation using a new model system. J Histochem Cytochem 33:229-239.

Jackson A, Crossman AR (1983) Nucleus tegmenti pedunculopontinus: efferent connections with special reference to the basal ganglia, studied in the rat by anterograde and retrograde transport of horseradish peroxidase. Neuroscience 10:725-765.

Jones BE (1990) Relationship of GABAergic to cholinergic neurons within the laterodorsal and pedunculopontine tegmental nuclei. Soc Neurosci Abstr 16:300.

Jones BE (1993) The organization of central cholinergic systems and their functional importance in sleep-waking states. Prog Brain Res 98:61-71.

Kharazia VN, Weinberg RJ (1994) Glutamate in thalamic fibers terminating in layer IV of primary sensory cortex. J Neurosci 14:60216032.

Kita H, Kitai ST (1987) Efferent projections of the subthalamic nucleus in the rat: light and electron microscopic analysis with the PHA-L method. J Comp Neurol 260:435-452.

Lavoie B, Parent A (1994a) Pedunculopontine nucleus in the squirrel monkey: projections to the basal ganglia as revealed by anterograde tract-tracing methods J Comp Neurol 344:210-231. 
I.avoie B, Parent A (1994b) Pedunculopontine nucleus in the squirrel monkey: distribution of cholinergic and monoaminergic neurons in the mesopontine tegmentum with evidence for the presence of glutamate in cholinergic neurons. J Comp Neurol 344:190-209.

Lavoie B, Parent A (1994c) Pedunculopontine nucleus in the squirrel monkey: cholinergic and glutamatergic projections to the substantia nigra. J Comp Neurol 344:232-241.

Lee HJ, Rye DB, Hallanger AE, Levey AI, Wainer BH (1988) Cholinergic vs noncholinergic efferents from the mesopontine tegmentum to the extrapyramidal motor system nuclei. J Comp Neurol 275:469492.

Levey AI, Edmunds SM, Heilman CJ, Desmond TJ, Frey KA (1994) Localization of muscarinic $m 3$ receptor protein and $m 3$ receptor binding in rat brain. Neuroscience 63:207-221.

Llewellyn-Smith IJ, Chalmers JP, Minson JB, Phend KD, Pilowsky M (1992) Glutamate-immunoreactive synapses on retrogradely-labeled sympathetic preganglionic neurons in rat thoracic spinal cord. Brain Res 581:67-80.

Matsumara M, Kojima J, Gardiner WT, Hikosaka O (1992) Visual and oculomotor functions of the monkey subthalamic nucleus. J Neurophysiol 67:1615-1632.

Mesulam M, Mash D, Hersh L, Bothwell M, Geula C (1992) Cholinergic innervation of the human striatum, globus pallidus, subthalamic nucleus, substantia nigra and red nucleus. J Comp Neurol 323:252268.

Miller WC, DeLong MR(1987) Altered tonic activity of neurons in the globus pallidus and subthalamic nucleus in the primate MPTP model of parkinsonism. Adv Behav Biol 32:415-427.

Mitchell IM, Boyce S, Sambrook MA, Crossman AR (1992) A 2-deoxyglucose study of the effects of dopamine agonists on the parkinsonian primate brain-implications for the neuronal mechanisms that mediate dopamine-agonist induced dyskinesia. Brain 115:809-825.

Moon Edley S, Graybiel AM (1983) The afferent and efferent connections of the feline nucleus tegmenti pedunculopontinus, pars compacta. J Comp Neurol 217:187-215.

Morizumi T, Nakamura Y, Kitao Y, Kudo M (1987) Ultrastructural andyses of afferent terminals in the subthalamic nucleus of the cat with a combined degeneration and horseradish peroxidase tracing method. J Comp Neurol 265:159-174

Nakanishi H, Kita H, Kitai ST (1987) Intracellular study of rat substantia nigra pars reticulata neurons in an in vitro slice preparation: electrical membrane properties and response characteristics to subthalamic stimulation. Brain Res 437:35-44.

Nakanishi, H, Kita H, Kitai ST (1991) Intracellular study of rat entopeduncular nucleus neurons in an in vitro slice preparation: response to subthalamic stimulation. Brain Res 549:285-291.

Nitsch C, Riesenberg R (1988) Immunocytochemical demonstration of GABAergic synaptic connections in the rat substantia nigna after dif ferent lesions of the striatonigral projection. Brain Res 461:127-142.

Nomura S, Noboru M, Tetsuo S (1980) Direct projections from the pedunculopontine tegmental nucleus to the subthalamic nucleus in the cat. Brain Res 196:223-227.

Ottersen OP (1989) Quantitative electron microscopic immunocytochemistry of neuroactive amino acids. Anat Embryol 180:1-15.

Ottersen OP, Storm-Mathisen J (1989) Excitatory and inhibitory amino acids in the hippocampus. In: The hippocampus: new vistas (ChanPalay V, Kohler C, eds). New York: Liss.

Paxinos G, Watson C (1986) The rat brain in stereotaxic coordinates. Sydney: Academic.

Petrusz P, Van Eyck SL, Weinberg RJ, Rustioni A (1990) Antibodies to glutamate and aspartate recognize non-endogenous ligands for excitatory amino acid receptors. Brain Res 529:339-344.

Phend KD, Weinberg RW, Rustioni A (1992) Techniques to optimise postembedding single and double staining for amino acid neurotransmitters. J Histochem Cytochem 40:1011-1020

Ribak CE, Vaughn JE, Saito K, Barber R, Roberts E (1976) Immu nocytochemical localization of glutamate decarboxylase in rat sub stantia nigra. Brain Res 1 16:287-298.

Rinvik E, Ottersen OP (1993) Terminals of subthalamonigral fibers are enriched with glutamate-like immunoreactivity: an electron micro scopic immunogold analysis in the cat. J Chem Neuroanat 6:19-30.

Rye DB, Saper CB, Lee HJ, Wainer BH (1987) Pedunculopontine tegmental nucleus of the rat: cytoarchitecture, cytochemistry, and some extrapyramidal connections of the mesopontine tegmentum. J Comp Neurol 259:483-528

Saper CB, Loewy AD (1982) Projections of the pedunculopontine tegmental nucleus in the rat: evidence for additional extrapyramidal circuitry. Brain Res 252:367-372.

Scarnati E, Proia A, Di Loreto S, Pacitti C (1987) The reciprocal electrophysiological influence between the nucleus tegmenti pedunculopontinus and the substantia nigra in normal and decorticated rats. Brain Res 423:116-124.

Smith Y, Parent A (1988) Neurons of the subthalamic nucleus in primates display glutamate but not GABA immunoreactivity. Brain Res $453: 353-356$

Smith Y, Bolam JP, von Krosigk M (1990a) Topographical and synaptic organization of the GABA-containing pallidosubthalamic projection in the rat. Eur J Neurosci 2:500-511.

Smith Y, Hazrati LN, Parent A (1990b) Efferent connections of the subthalamic nucleus in the squirrel monkey as studied by the PHA-L anterograde tracing method. J Comp Neurol 294:306-323.

Smith Y, Wichmann T, DeLong MR (1994a) Analysis of the synaptic innervation of neurones in the internal pallidal segment by the sub thalamic nucleus and the external pallidum in monkey. J Comp Neurol 343:297-318.

Smith Y, Charara A, Parent A (1994b) Differential synaptic innervation of dopaminergic neurones in the ventral tegmental area and the substantia nigra pars compacta by glutamate-containing terminals in the squirrel monkey. Mov Disord 9(Suppl) 1:110.

Somogyi P, Hodgson AJ (1985) Antiserum to $\gamma$-aminobutyric acid. III Demonstration of GABA in Golgi-impregnated neurons and in conventional electron microscopic sections of cat striate cortex. J His tochem Cytochem 33:249-257.

Somogyi P, Hodgson AJ, Chubb IW, Penke B, Erdei A (1985) Antisera to $y$-aminobutyric acid. II. Immunocytochemical application to the central nervous system. J Histochem Cytochem 33:240-248.

Somogyi P, Halasy K. Somogyi J, Storm-Mathisen J, Ottersen OP (1986) Quantification of immunogold labeling reveals enrichment of glutamate in mossy and parallel fiber terminals in cat cerebellum. Neuroscience 19:1045-1050.

Spann BM, Grofova I (1989) Origin of ascending and spinal pathways from the nucleus tegmenti pedunculopontinus in the rat. $\mathrm{J}$ Comp Neurol 283:13-27.

Sugimoto T, Hattori T (1984) Organization and efferent projections of nucleus tegmenti pedunculopontinus pars compacta (TPC) with spe cial reference to its cholinergic aspects. Neuroscience 11:931-946.

Tremblay L, Filion M (1989) Responses of pallidal neurons to striatal stimulation in intact waking monkeys. Brain Res : 1-16.

Valtschanoff JG, Phend KD, Bernadi PS, Weinberg RJ, Rustioni A (1994) Amino acid immunocytochemistry of primary afferent terminals in the rat dorsal horn. J Comp Neurol 346:237-252.

Veenman CL, Reiner A, Honig MG (1992) Biotinylated dextran amine as an anterograde tracer for single- and double labeling studies. J Neurosci Methods 41:239-254.

Vincent SR, Satoh K, Armstrong DM, Fibiger HC (1983) NADPHdiaphorase: a selective histochemical marker for the cholinergic neurons of the pontine reticular formation. Neurosci Lett 43:31-36.

von Krosigk M, Smith AD (1991) Descending projections from the substantia nigra and the retrorubral field to the medullary and pontomedullary reticular formation. Eur J Neurosci 3:260-273.

Wichmann T, Bergman H, DeLong MR (1994a) The primate subthalamic nucleus. I. Functional properties in intact animals. J Neurophysiol 72:494-506

Wichmann T, Bergman H, DeLong MR (1994b) The primate subthalamic nucleus. III. Changes in motor behaviour and neuronal activity in the internal pallidum induced by subthalamic inactivation in the MPTP model of parkinsonism. J Neurophysiol 72:521-530.

Woolf NJ, Butcher LL (1986) Cholinergic systems in the rat brain. III Projections from the pontomesencephalic tegmentum to the thalamus, tectum, basal ganglia, and basal forebrain. Brain Res Bull 16:603663. 